ARTICLE

\title{
Structural basis of prokaryotic ubiquitin-like protein engagement and translocation by the mycobacterial Mpa-proteasome complex
}

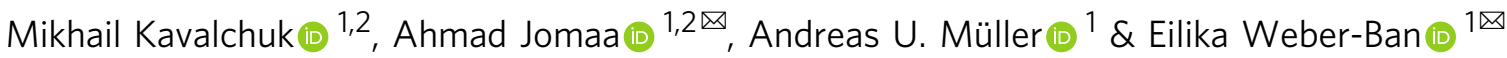

Proteasomes are present in eukaryotes, archaea and Actinobacteria, including the human pathogen Mycobacterium tuberculosis, where proteasomal degradation supports persistence inside the host. In mycobacteria and other members of Actinobacteria, prokaryotic ubiquitinlike protein (Pup) serves as a degradation tag post-translationally conjugated to target proteins for their recruitment to the mycobacterial proteasome ATPase (Mpa). Here, we use single-particle cryo-electron microscopy to determine the structure of Mpa in complex with the $20 \mathrm{~S}$ core particle at an early stage of pupylated substrate recruitment, shedding light on the mechanism of substrate translocation. Two conformational states of Mpa show how substrate is translocated stepwise towards the degradation chamber of the proteasome core particle. We also demonstrate, in vitro and in vivo, the importance of a structural feature in Mpa that allows formation of alternating charge-complementary interactions with the proteasome resulting in radial, rail-guided movements during the ATPase conformational cycle. 
E nergy-dependent protein degradation contributes to cellular protein quality control and general protein turnover, and ensures a dynamic proteome response to changing cellular states and environments $s^{1,2}$. It is carried out by compartmentalizing degradation machines consisting of a proteolytic core cylinder that must associate with ring-shaped, ATP-dependent regulator complexes in order to recruit, unfold and translocate its target protein substrates for processive degradation ${ }^{3}$. In eukaryotes, this activity is carried out by the $26 \mathrm{~S}$ proteasome made of the $20 \mathrm{~S}$ core particle (CP) and the $19 \mathrm{~S}$ regulatory particle, which recruits proteins post-translationally modified with polyubiquitin (poly-Ub) chains ${ }^{4}$. Bacteria usually do not have proteasomes but possess complexes of related, modular architecture such as the Clp-protease complexes and HslUV. Interestingly, mycobacteria and other members of Actinobacteria have in addition obtained $20 \mathrm{~S}$ proteasome subunits by horizontal gene transfer ${ }^{5,6}$. The bacterial $20 \mathrm{~S}$ proteasome interacts with a ring-shaped AAA regulatory complex called the mycobacterial proteasome ATPase (Mpa) in mycobacteria or ARC (ATPase forming ring-shaped complexes) in other actinobacterial species ${ }^{7-10}$. Furthermore, these bacteria evolved a unique substrate tagging and proteasome delivery pathway that is functionally analogous to ubiquitination ${ }^{11,12}$. The prokaryotic ubiquitin-like protein Pup is post-translationally ligated to lysine side chains of substrate proteins by the Pup ligase PafA, which marks them for degradation by the Mpa-20S CP complex $^{13,14}$. The resulting isopeptide linkage is formed between the sidechain carboxylate of the C-terminal glutamate residue of Pup and the substrate lysine ${ }^{15}$. Pupylation can be reversed by the depupylase Dop that catalyzes the cleavage of the isopeptide bond between Pup and substrate proteins ${ }^{16,17}$. Unlike ubiquitin, Pup is intrinsically disordered in its free state ${ }^{18-20}$ or when covalently attached to substrate proteins ${ }^{21}$. However, upon binding to the pupylation/depupylation enzymes, it forms two orthogonal helices in the C-terminal half of its primary sequence, fitting closely into the 40-50 $\AA$-long Pup-binding groove present on Dop and PafA ${ }^{22}$. Pupylation and proteasomal degradation were shown to ensure survival of the bacteria under challenging conditions like during persistence of Mycobacterium tuberculosis (Mtb) inside host macrophages, under nitrogenstarvation or under DNA-damaging conditions ${ }^{23-27}$.

$\mathrm{Mpa} / \mathrm{ARC}$ is related in architecture and domain structure to the six eukaryotic proteasomal ATPase subunits Rpt1-6 present in the $19 \mathrm{~S}$ regulatory particle ${ }^{9}$. The protomer features the canonical AAA module, preceded by two consecutive $\beta$-barrel-shaped oligonucleotide-binding $(\mathrm{OB})$ domains and an N-terminal $\alpha$ helical domain ${ }^{28}$. In the hexameric ring, the N-terminal helices form a total of three coiled-coils by interacting with their respective neighboring $\mathrm{N}$-terminal $\alpha$-helical domains. The three coiled-coils emanate from the stiff flange-like structure formed by the six double-OB domains. While in the eukaryotic 19S regulatory particle the coiled-coil domains mediate binding of nonATPase subunits (Rpn) and the positioning of ubiquitin receptors ${ }^{29,30}$, they constitute the direct substrate recruitment domains in $\mathrm{Mpa} / \mathrm{ARC}^{19}$. Pup undergoes another disorder-toorder transition, when binding to the Mpa coiled-coil domains, forming a single long helix of 30 residues (21-51 in $\mathrm{Mtb}$ ) that engages into an antiparallel shared coiled-coil interaction with the Mpa N-terminal coiled-coil domains ${ }^{19,31}$. Due to the antiparallel arrangement of the interaction with $\mathrm{Mpa}$, the $\mathrm{N}$-terminal region of Pup points toward the Mpa pore. Interestingly, the N-terminal segment of Pup remains disordered and acts as a threader sequence for Mpa pore engagement ${ }^{8,32}$. Subsequent unfolding and translocation is mediated by Mpa pore loops carrying the canonical Ar-Hb-Gly motif (F341-V342-G343 in Mtb) conserved in AAA family members ${ }^{8}$.
Binding of Mpa to the proteasome core is mediated via a C-terminal GQYL motif, featuring a penultimate tyrosine ${ }^{8,33}$, similar to the eukaryotic 19S ATPase subunits that employ the conserved C-terminal HbYX motif ${ }^{34,35}$. Stable interaction and consequently efficient degradation of pupylated substrates by the Mpa-proteasome of Mtb in vitro could only be observed with an open-gate variant lacking the $\mathrm{N}$-terminal seven residues on the $\alpha$-subunits, even though the full-length core particle is active in vivo ${ }^{36}$. An X-ray structure of the Mpa hexamer in the absence of the 20S proteasome and without substrate suggested that due to a stable $\beta$-grasp domain preceding the $\mathrm{C}$-terminal proteasome-interaction motif, the C-termini are located inside the Mpa channel and are unavailable for interaction with the proteasome ${ }^{28}$. However, cryo-electron microscopic analysis of a partially resolved Mpa-hexamer suggested that this region might show some conformational flexibility ${ }^{37}$.

Despite the importance of the Mtb proteasome as a virulence factor for one of the most successful human pathogens ${ }^{27,38,39}$, the structural and biochemical information on its interaction with the AAA unfoldase Mpa, responsible for recruitment of pupylated substrates, is still limited.

Here, we utilize single-particle cryo-electron microscopy (cryo-EM) to determine the structure of the Mpa-proteasome complex at the stage of Pup translocation into the Mpa pore. We resolved two distinct conformational states corresponding to different stages during substrate translocation by the hexameric ATPase ring. The spiral-staircase arrangement of the Mpa pore loops around the translocating substrate chain is in agreement with the canonical AAA+ substrate unfolding mechanism. Interestingly, our structure suggests that, in spite of considerable changes in the Mpa conformation taking place during substrate translocation, $\mathrm{Mpa}$ interactions with the proteasome are maintained through the formation of a series of chargecomplementary contacts along radially distributed rail-like features of the proteasome core particle. The importance of these interactions for the Mpa-proteasome substrate translocation/ degradation mechanism is further supported by in vitro biochemical data as well as in vivo substrate degradation.

\section{Results}

Generation of a substrate-engaged Mpa-proteasome complex for structural analysis. In order to obtain a molecular understanding of the interactions between $\mathrm{Mpa}$ and the $20 \mathrm{~S}$ proteasome as well as mechanistic insights into the engagement of pupylated substrates into the Mpa pore, we aimed to generate a substrate-engaged Mpa-20S CP complex for study by cryo-EM. To favor complex formation, seven residues were N-terminally deleted from the proteasomal $\alpha$-subunits $(\triangle 7 \operatorname{PrcA})$, as this was shown to stabilize the interaction ${ }^{7}$. A homogeneously Pupdecorated model substrate was generated by placing the Pup coding sequence in-frame upstream of the gene folA encoding dihydrofolate reductase (DHFR), resulting in a linear PupDHFR fusion, in which the Pup C-terminal residue is ligated to the DHFR amino terminus through a regular peptide bond (Fig. 1a). It has been demonstrated previously that linear Pup-substrate fusions can be recruited and degraded by the Mpa-proteasome complex ${ }^{8}$. As expected, PupDHFR is degraded by the Mpa-20S CP complex in the presence of ATP. Under the chosen conditions at $37^{\circ} \mathrm{C}$, PupDHFR was fully degraded within $30 \mathrm{~min}$ after adding ATP (Fig. 1b, upper gel). When the reaction is carried out at $4{ }^{\circ} \mathrm{C}$ with the addition of ATP $\gamma \mathrm{S}$ after 1 min of incubation, no discernable amount of PupDHFR is degraded in the same time frame (Fig. 1b, lower gel), suggesting that substrate is stalled at the stage of Mpa translocation, analogously to what was observed for the human $26 \mathrm{~S}$ proteasome engaged with a 
a

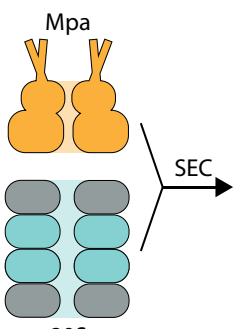

$20 \mathrm{~S}$

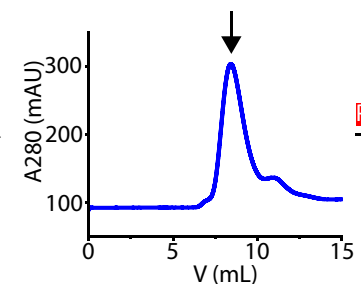

$(\mathrm{mL})$

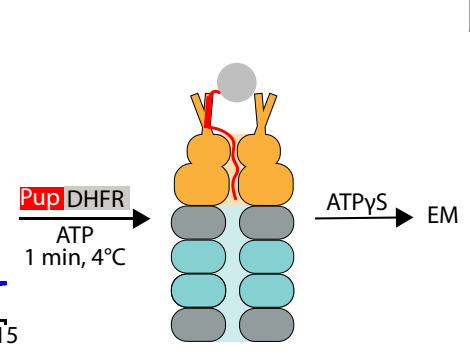

b

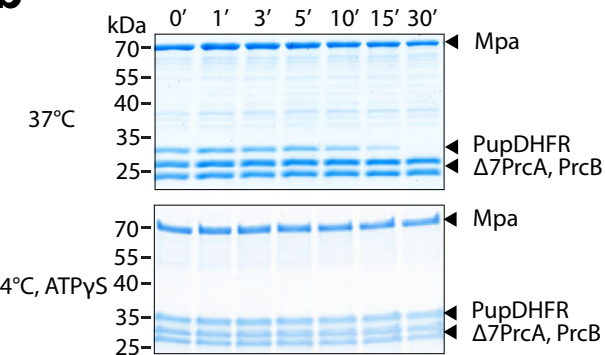

c

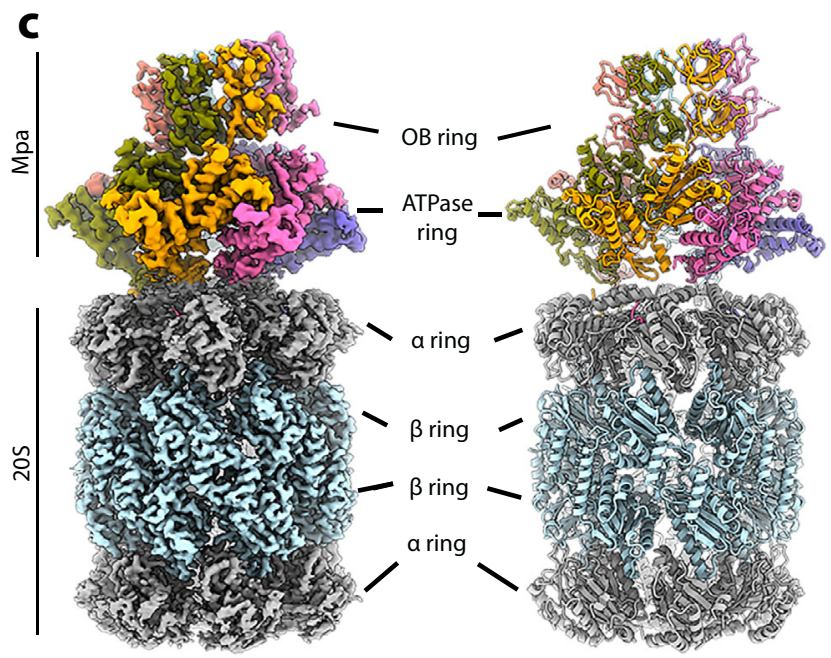

d

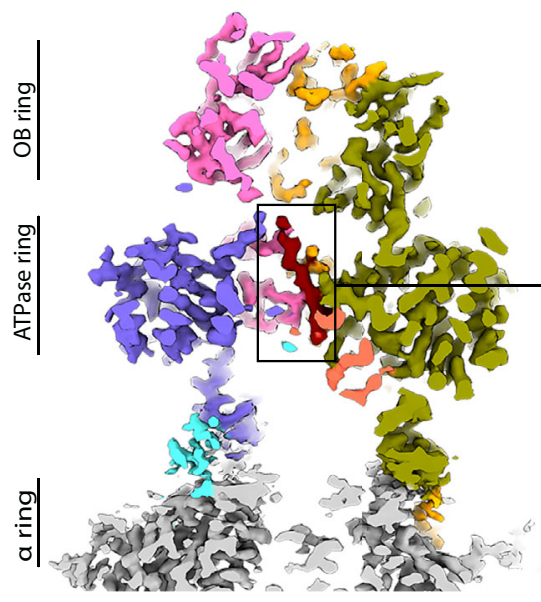

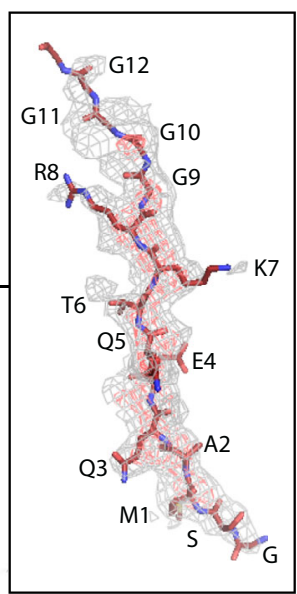

Fig. 1 Generation and structure determination of a substrate-engaged Mpa-20S proteasome complex from Mycobacterium tuberculosis. a Cartoon depicting generation of the stalled, substrate-engaged Mpa-20S complex. Open-gate 20S core particle (gray and light blue), Mpa (orange) and ATP were incubated together and the mixture was subjected to size-exclusion chromatography (SEC; the SEC profile image was generated using Origin 2018). The Mpa-20S core particle elution peak, pupylated model substrate (linear Pup-dihydrofolate reductase fusion PupDHFR) and ATP were incubated for 60 s on ice. After addition of ATP $\gamma$ S, the sample was immediately applied onto the EM grid. $\mathbf{b}$ PupDHFR degradation was monitored by Coomassie-stained SDSPAGE. The degradation reaction containing open-gate proteasome ( $\triangle 7 \mathrm{PrcA}, \operatorname{PrcB}$ ), wild-type Mpa and PupDHFR was started by addition of ATP and was carried out at $37^{\circ} \mathrm{C}$ (upper gel) and at $4{ }^{\circ} \mathrm{C}$ with the addition of ATP $\gamma$ S after 1 min (lower gel). Aliquots for SDS-PAGE analysis were taken at the indicated time points. Representatives of three individual experiments are shown. Source Data are provided as a Source Data file. c Composite cryo-EM map of the $20 \mathrm{~S}$ proteasome and substrate-engaged $\mathrm{Mpa}$ in state A after 3D image classification and focused 3D refinement. Cryo-EM densities of 20S $\alpha$ and $\beta$ subunits are colored gray and light blue, respectively. Mpa protomes densities representing oligonucleotide-binding (OB) and ATPase domains are colored pink, blue, cyan, yellow, orange, and green. Pup is colored red. $\mathbf{d}$ Cross-section of the cryo-EM density of the $20 \mathrm{~S} \alpha$-ring gate (gray) and Mpa engaged with the $\mathrm{N}$-terminal Pup segment inside the AAA chamber. EM density corresponding to the first 12 residues of Pup inside the Mpa ATPase chamber is shown with fitted atomic coordinates.

ubiquitinated substrate ${ }^{40}$. Therefore, to prepare the sample for electron microscopy, we assembled the Mpa-20S complex in the presence of ATP, and added the pupylated substrate while keeping the sample on ice to allow for substrate translocation and slow ATP turnover. The reaction was quenched with ATP $\gamma \mathrm{S}$ to prevent further cycles of ATP hydrolysis and to stall substrate translocation, before application to the electron microscopy grid and vitrification (Fig. 1a) ${ }^{40}$.

Overall architecture of the substrate-engaged Mpa-proteasome complex reveals two Mpa states. We originally picked 860,718 particles for 2D classification, presenting both top and side views of the proteasome, of which 385,394 were used for 3D classification (Supplementary Fig. 1). The initial 2D and 3D image processing scheme visualized a class with Mpa-bound proteasome in addition to a class containing uncapped 20S CP. The structure of Mpa bound to the 20S CP that was determined without applying any symmetry, revealed a 6-edged, star-shaped density originating from a hexameric Mpa bound to the heptameric $\alpha$-ring of the 20S CP. To improve the local resolution of
Mpa, we performed local 3D classification and 3D refinement combined with signal subtraction of the 20S CP (see "Methods"). This approach resolved two states of Mpa at an average resolution of $3.8 \AA$ and $3.9 \AA$, respectively (Supplementary Fig. 2). The two states correspond to two distinct motor conformations stalled at sequential stages of substrate translocation. The structure of the 20S CP particle was determined by focused refinement using a binary mask for this region and was resolved at $2.8 \AA ̊$ resolution (Supplementary Fig. 1, Supplementary Fig. 2, Supplementary Table 1).

The cryo-EM structures of the Mpa-20S CP complex reveal the architecture of a co-axially aligned AAA-protease assembly featuring a substrate conduit from the entrance pores of the slightly tilted Mpa tandem $\mathrm{OB}$ domains to the AAA motor and into the $\alpha$-ring of the $20 \mathrm{~S} \mathrm{CP}$ (Fig. 1c, d). The protomers of the Mpa hexamer assemble in a spiral staircase, an arrangement that has emerged as a hallmark of AAA translocation motors ${ }^{41}$ (Fig. 1d; Supplementary Fig. 3). States A and B exhibit several differences including a varying degree of upward rotation $\left(\sim 30^{\circ}\right)$ of the double-OB ring with respect to the AAA motor (Fig. 2, side views). A long loop serves as a flexible connection that can 
a
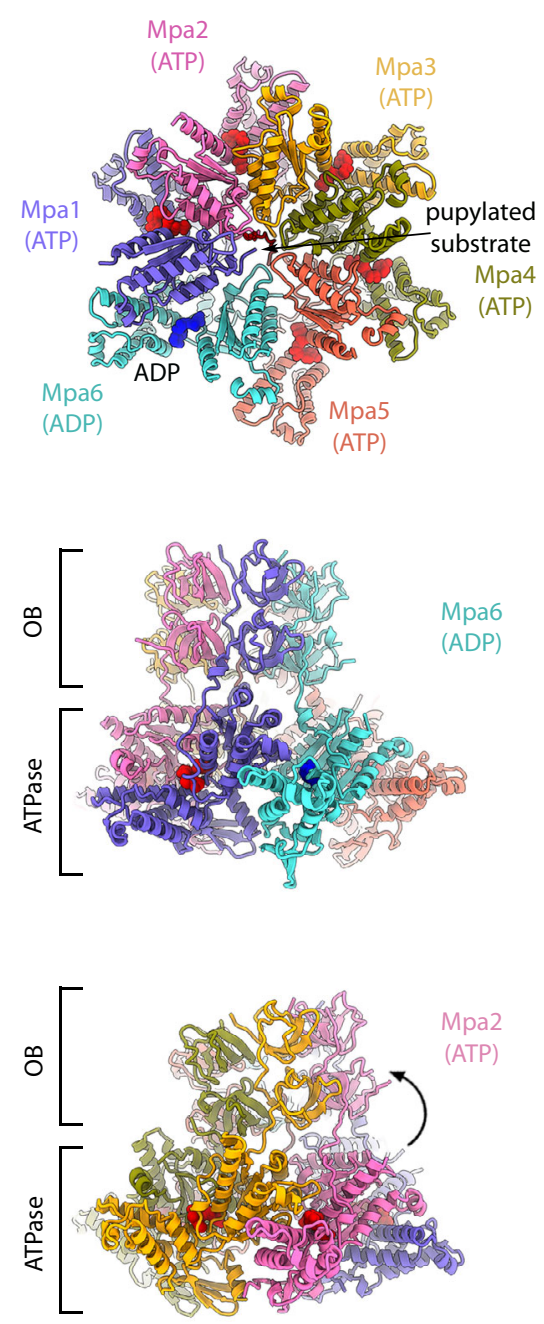

20S·Mpa·PupDHFR state A b
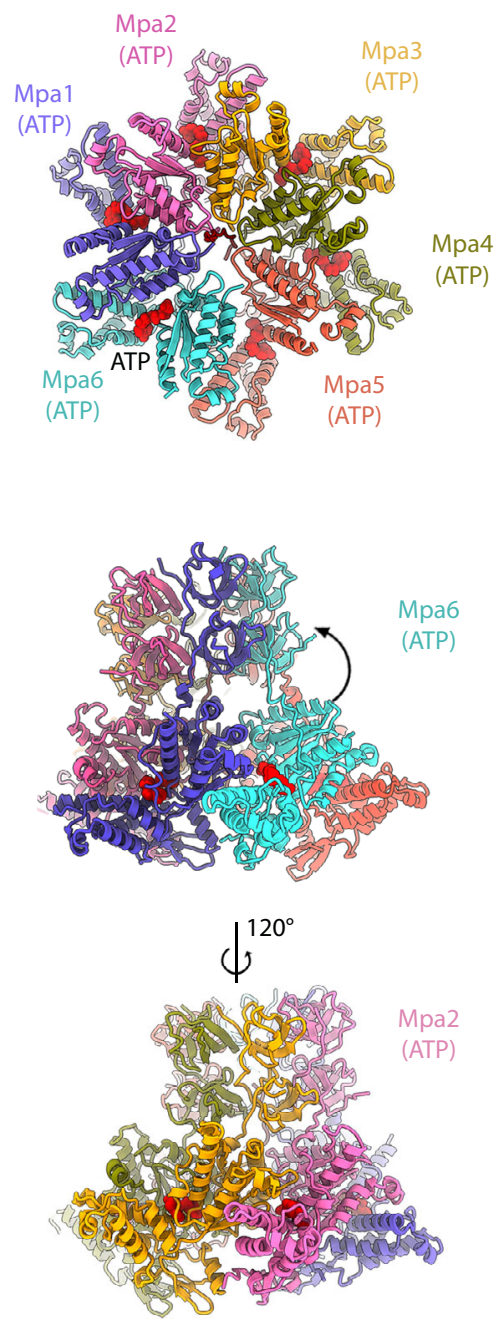

20S·Mpa·PupDHFR

state $B$

Fig. 2 Mycobacterial AAA unfoldase Mpa engaged with PupDHFR substrate presents in two conformational states. a, $\mathbf{b}$ Coordinates of Mpa in state $A$ and $B$ are shown in cartoon representation in top view (upper) and side view (middle and lower). ATP and ADP molecules are shown as spheres and are colored red and blue, respectively. Mpa protomers (Mpa1-6) and substrate are colored with the same color code as used in Fig. 1. The conformational change in the OB-ring is indicated by an arrow.

dynamically accommodate the tilt and movements in the AAA motor during substrate processing. In state $\mathrm{A}$, the largest rotation was resolved at protomer 2 (pink) site, while in state $B$, the double-OB-ring displays the largest tilt at protomer 6 (cyan) site.

Inside the ATPase chamber of the Mpa-20S CP complex in both states, a tubular density is resolved engaged with the ATPase pore loops of the Mpa protomers (Fig. 1d; Supplementary Fig. 3). Based on the visible side-chain density, this tubular EM-density can be assigned to the first 15 residues of the Mtb Pup sequence (Fig. 1d). An additional Ser-Gly motif N-terminal of the first Pup residue is left over after TEV protease cleavage and is also resolved in the Mpa channel. The EM density of the engaged substrate becomes weaker following the Pup sequence out of the AAA chamber toward the double-OB ring of Mpa. The amino acid sequence of this region of Pup features five consecutive glycines and four aspartates, the latter usually lacking side-chain density in cryo-EM maps ${ }^{42}$. Substrate could not be further visualized beyond the $\mathrm{OB}$ rings, indicating greater conformational heterogeneity of Pup in that region. The coiled-coil N-terminal helices of Mpa that emanate from the surface of the OB ring and first recognize pupylated substrates ${ }^{19,31}$ are expected to be flexible and were not visualized. On the side of the proteasome, the substrate density approaches the open gate but it does not yet enter the degradation chamber (approximately $35 \AA$ distance from the first resolved residue to the gate). Therefore, the complexes trapped in our study reveal the initial events of substrate engagement by the AAA motor prior to translocation into the proteasome for degradation.

Mpa associates with the $20 \mathrm{~S}$ proteasome via its C-terminal GQYL motif and the $\beta$-hairpin loops of the $\beta$-grasp domains. Proteasome regulatory ATPases are known to feature a C-terminal motif with a penultimate tyrosine residue as the main binding determinant for association with the $20 \mathrm{~S}$ core particles to form the proteasome holocomplexes in both eukaryotes and bacteria $^{8,35}$. A structure of Mpa determined in the absence of the proteasome showed the C-termini pointing back into the pore, 
a

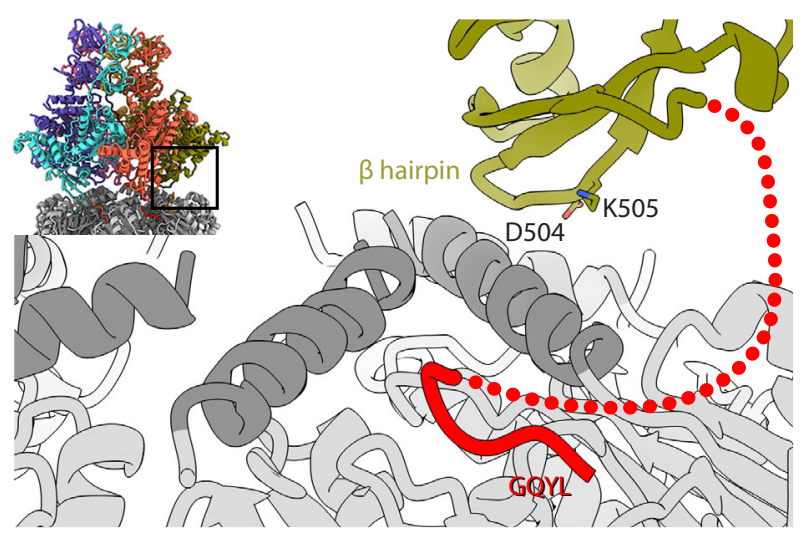

C

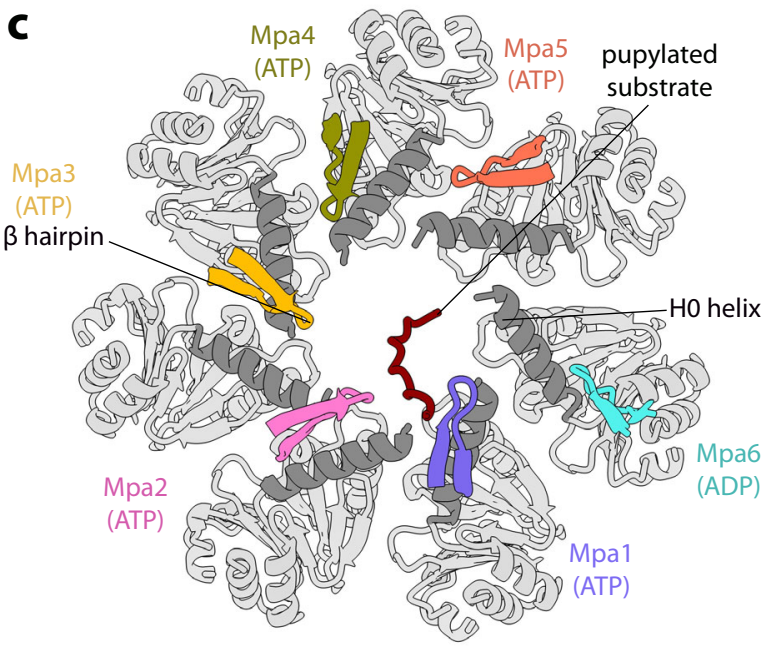

20S.Mpa.PUPDHFR state $\mathrm{A}$

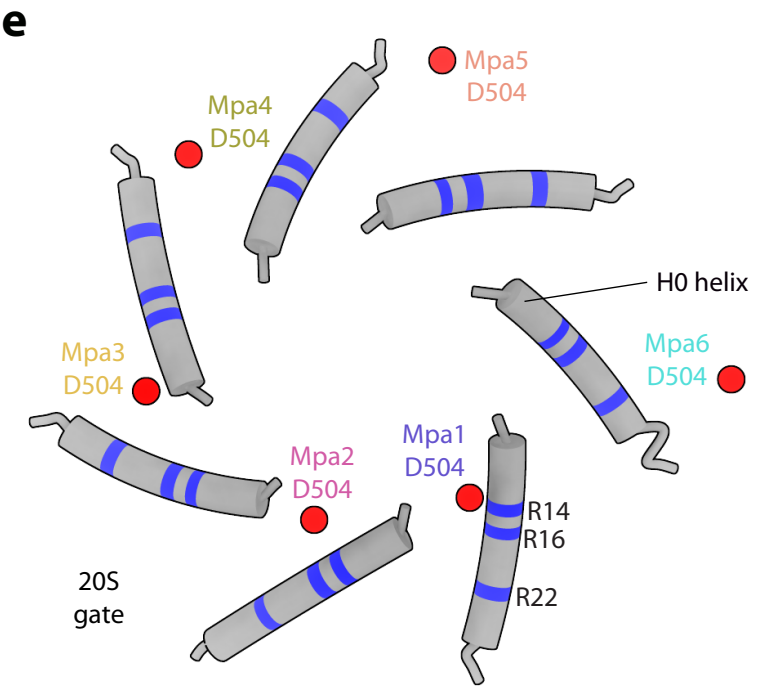

State A

presumably unavailable for interaction, although the GQYL motif itself was not resolved ${ }^{28}$. Our structure of the substrate-engaged proteasome resolves the interaction between $\mathrm{Mpa}$ and the gate of the 20S CP (Fig. 3a). It demonstrates that the C-terminal extensions of Mpa containing the terminal GQYL motifs are able to adopt a conformation facing outside of the Mpa pore, and that their GQYL motifs occupy the binding pockets between the b

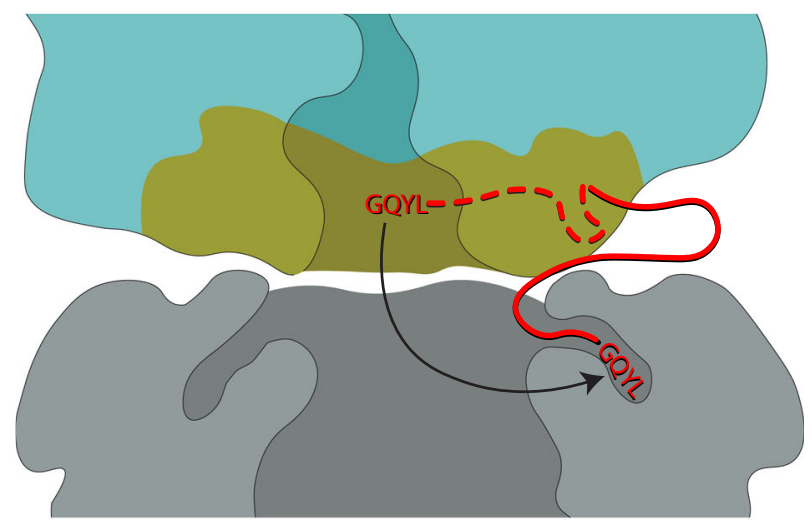

d

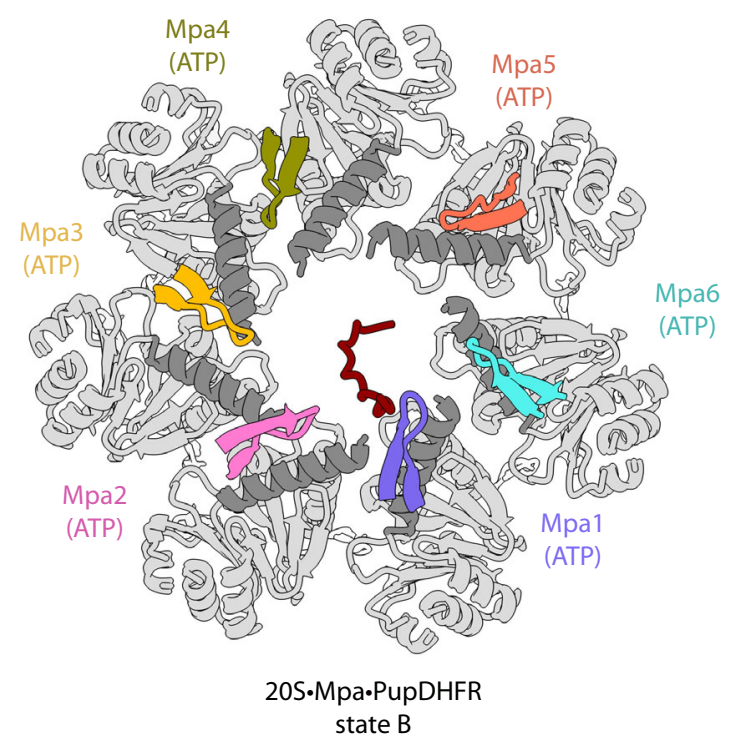

f

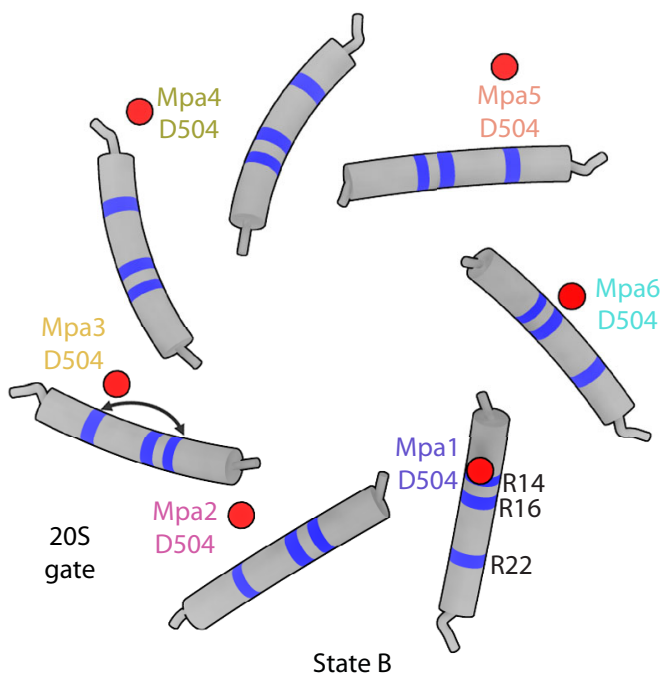

proteasome $\alpha$-ring subunits upon complex formation (Fig. $3 \mathrm{~b}$ and Supplementary Fig. 4). Although the $\alpha$-ring subunits lack the $\mathrm{N}$-terminal seven residues in the open-gate $20 \mathrm{~S} \mathrm{CP}$, we still observed a slight increase in proteasomal gate diameter on the Mpa-bound side compared to the Mpa-free side, which is indicative of the conformational change undergone by the proteasomal $\alpha$-rings upon insertion of the GQYL motifs into the $\alpha$-ring 
Fig. 3 Interaction of Mpa with the proteasome $\boldsymbol{\alpha}$-rings. a Close-up of the Mpa/20S $\alpha$-ring interface including the C-terminal extension of one Mpa protomer with the GQYL proteasome-interaction motif. $\mathrm{HO}$ helices are colored dark gray. The dashed red line indicates the flexible fragment connecting the Mpa $\beta$-grasp domain with the GQYL C-terminal motif. Charged $\beta$-hairpin residues D504 and K505 are shown as sticks and labeled. b The Mpa $\beta$-grasp domain (olive) adopts a different conformation upon binding to the proteasome (gray), releasing the C-terminal GQYL motif (red) and allowing it to bind into the $\alpha$-ring binding pockets (dark gray). c Close-up top view of the proteasome gate shown as cartoon and colored in light gray. The $\mathrm{N}$-terminal $\mathrm{HO}$ helices of the proteasome $\alpha$-ring protomers are colored darker gray. The $\beta$-hairpin loops of Mpa (state A) are colored according to the color scheme used in Fig. 2. $\mathbf{d}$ Same as (c) except that the conformations of the $\beta$-hairpin loops for each of the Mpa protomers are shown as observed in state B. e, $\mathbf{f}$ Schematic representation of the proteasome $\mathrm{N}$-terminal $\mathrm{HO}$ helices shown as gray cylinders and $\beta$-hairpin loop residue D504 shown as a red dot. Chargecomplementary contact residues (R14, R16, R22) on the proteasome HO helices are marked as blue stripes. The sliding of the contacting residue (D504) in a rail-like motion on the $\mathrm{N}$-terminal helices of the $20 \mathrm{~S}$ proteasome gate is indicated by an arrow.

binding pockets (Supplementary Fig. 5a, b). Densities for the GQYL motif were resolved in all seven pockets due to averaging resulting from the inherent D7 symmetry of the 20S CP (Supplementary Fig. 4). We tentatively assigned six GQYL motifs based on their proximity to the respective Mpa protomers. The last GQYL motif was not assigned to any of the Mpa protomers and was included as a separate chain in the model.

Interestingly, our structure reveals that the $\beta$-grasp domains of Mpa contribute a second contact in the form of the $\beta$-hairpin loop between strands 16 and 17, which interacts with the $\mathrm{N}$-terminal H0 helices that form the gate of the CP (Fig. 3a, c, d). These $\beta$-hairpin loops possess two charged (D504 and K505) residues, while the $\mathrm{N}$-terminal $\mathrm{H} 0$ helices of the core particle $\alpha$ subunits possess several glutamate (E10, E15, E18) and arginine (R14, R16, R22) residues, resulting in multiple points of possible electrostatic interactions for the hairpins along the helices. The array of charged residues along the H0 helices could serve as a "rail" for the $\beta$-hairpin loops that could move along the length of the $\mathrm{HO}$ helices by forming different combinations of transient electrostatic interactions based on proximity assessment (Fig. 3e, f). In state $A, M p a$ protomer 6 (cyan) depicts the $\beta$-hairpin conformation that is the farthest away from the gate pore in the outward position, while protomer 1 (blue) displays the most inward position right above the pore of the $\mathrm{CP}$ gate (Fig. $3 \mathrm{c}$, e; Supplementary Fig. 5c). These positions are slightly offset in state B (Fig. 3d, f; Supplementary Fig. 5d). While these interactions alone are not sufficient for the formation of a stable complex ${ }^{8}$, they likely serve to align the substrate conduit of Mpa with the gate of the CP despite the symmetry mismatch and the nucleotide-state-dependent conformational dynamics of the AAA motor.

To investigate how the $\beta$-hairpin loop interactions affect substrate degradation, an $\mathrm{Mpa}$ variant $\left(\mathrm{Mpa} \mathrm{HL}{ }^{\mathrm{Cg}}\right)$ was generated replacing hairpin residues 501-505 (ANGDK) with the equivalent but not conserved sequence stretch (IDGSV) from Corynebacterium glutamicum Mpa, where these interactions are absent, since C. glutamicum belongs to the group of Actinobacteria lacking the 20S CP (Supplementary Fig. 6). Additionally, a proteasome variant (H0-3RA) was generated where R14, R16 and R22 of the $\mathrm{H} 0$ helix were replaced with alanines. Proteasomal degradation of the model substrate PupDHFR mediated by either wild-type Mpa or the Mpa $\mathrm{HL}^{\mathrm{Cg}}$ variant in complex with open-gate 20S CP $(\triangle 7 \mathrm{CP})$ as well as by wild-type $\mathrm{Mpa}$ in complex with the proteasome variant $\triangle 7 \mathrm{CP} \_H 0-3 \mathrm{RA}$ was monitored by Coomassie-stained SDS-polyacrylamide gel electrophoresis (Fig. 4a). While in the presence of wild-type Mpa, PupDHFR was completely degraded within $15 \mathrm{~min}$ under the chosen conditions, Mpa HL ${ }^{\mathrm{Cg}}$ still had not completed the reaction in four hours, indicating more than an order of magnitude change in the degradation rate. A similar effect on PupDHFR degradation was observed in the presence of wild-type Mpa in complex with the proteasome variant $\triangle 7 \mathrm{CP} \_\mathrm{H} 0-3 \mathrm{RA}$.To ensure that $\mathrm{Mpa}$ $\mathrm{HL}^{\mathrm{Cg}}$ has no defect in ATPase activity, ATP hydrolysis was followed spectroscopically by coupling the production of inorganic phosphate to the turnover of 7-methylinosine to hypoxanthine by the enzyme purine nucleotide phosphorylase. No reduction in ATP hydrolysis rate but rather an increase was observed for Mpa HLCg $\left(237 \pm 6 \mathrm{~min}^{-1}\right)$ compared to Mpa WT $\left(139 \pm 1 \mathrm{~min}^{-1}\right)$, excluding the possibility that the impaired ability to deliver substrates to the CP for degradation stems from a defect in ATPase activity.

To assess the effect of the $\beta$-hairpin loop in vivo, we generated a Mycobacterium smegmatis $m p a$ knockout strain $(\Delta m p a)$ and complemented it with either wild-type Mpa or the $\beta$-hairpin variant expressed under control of the natural mpa promoter. The strains were transiently exposed to the DNA-damaging reagent mitomycin $\mathrm{C}$, resulting first in upregulation of recombinase $\mathrm{A}$ (RecA), a natural substrate of the Pup-proteasome system, followed by proteasomal degradation during the recovery phase from DNA damage stress ${ }^{25}$. RecA immunoblotting showed that the $\Delta m p a$ strain complemented with the Mpa HLCg $\beta$-hairpin loop variant exhibited slower RecA degradation compared to the strain complemented with the wild-type protein (Fig. 4b). Taken together, our findings support a role of the $\beta$-hairpin loop in mediating dynamic contacts between $\mathrm{Mpa}$ and the $\mathrm{CP}$ during pupylated substrate degradation.

Substrate is arrested in Mpa in the pore-engaged pre-handover state. Our cryo-EM structure captures the Mpa-proteasome complex at the initial stage of substrate translocation, where the Mpa pore channel is occupied with the N-terminal segment of Pup (Fig. 5a). We could reliably model the first 15 amino acids of the Pup sequence into the electron density occupying the Mpa channel, indicating that the Mpa-20S CP complexes are uniformly occupied with this stretch of sequence (Fig. 5b). Both of the resolved states (state $\mathrm{A}$ and $\mathrm{B}$ ) of the complex represent this early Pup-engagement intermediate (Supplementary Fig. 7a-c). A stacked pair of residues, the canonical aromatic pore loop residue F341 and the highly conserved adjacent lysine residue K340, forms a spiral-staircase that encircles the Pup N-terminal segment by engaging in unspecific interactions with its $\mathrm{Ca}$ backbone (Fig. 5c-e). Another channel loop about 16-17 $\AA$ further down (V384-S385-S386) forms a second spiral-staircase (Fig. 5b). It is resolved only in four of the six protomers, the other loops are flexible, as the Pup N-terminal segment has not yet reached far enough down at this state of engagement.

Interestingly, while in state $\mathrm{A}$, all six of the canonical aromatic pore loops of the ATPase domain are in close proximity to the substrate backbone with one of them (protomer 6, cyan) initially disengaged (Fig. $5 \mathrm{c}$ ), in state B only five protomers are in close proximity to the substrate backbone and engaged in interactions with it (Fig. 5d). The loop of the penultimate protomer (protomer 6 , cyan), which was at the bottom of the staircase in state A, is retracted away from the substrate in state $B$ and has shifted upward by $25 \AA$ to a more distal position relative to the proteasome gate (Fig. $5 c-e$ ). Furthermore, in state $B$, the five 


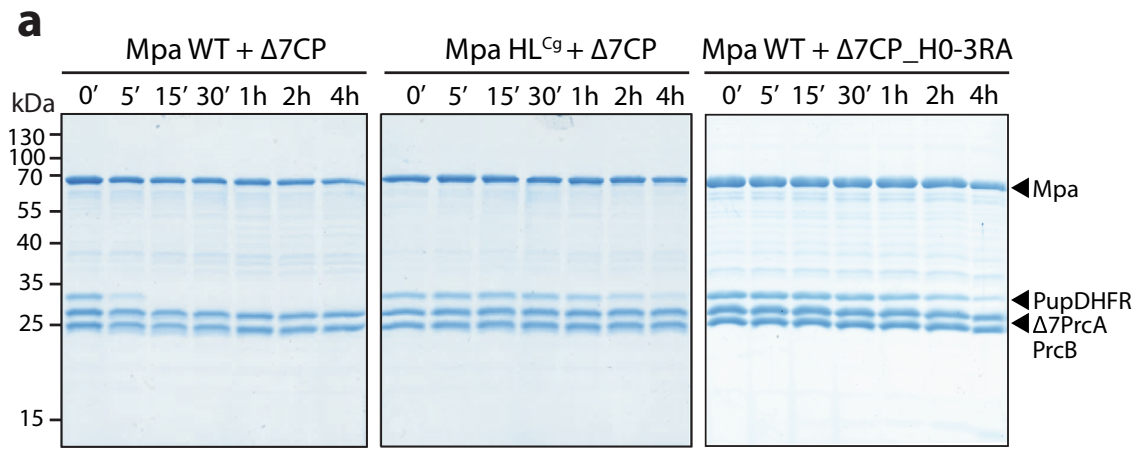

b

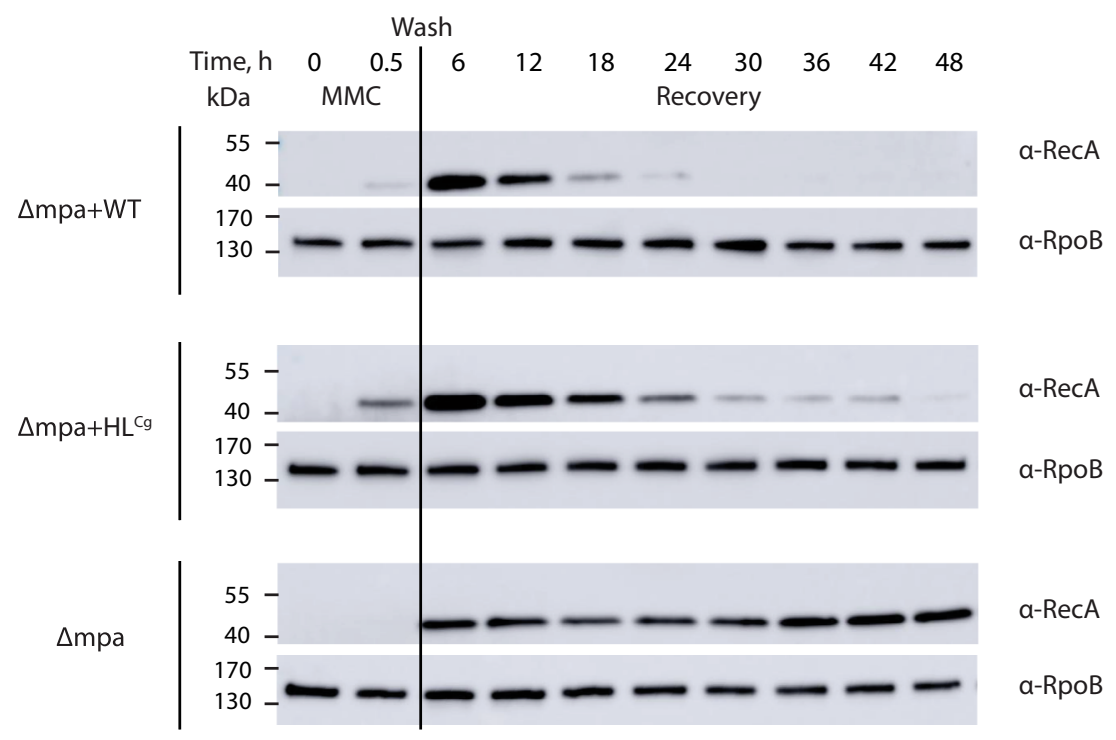

Fig. 4 Mutation of the Mpa $\boldsymbol{\beta}$-hairpin loop affects substrate degradation in vivo and in vitro. a Proteasomal degradation of PupDHFR mediated by wildtype $\mathrm{Mpa}$ (left) or $\mathrm{Mpa} \mathrm{HLCg}$ (middle) in complex with open-gate $20 \mathrm{~S} \mathrm{CP}\left(\Delta 7 \mathrm{CP}\right.$ ) and of wild-type Mpa in complex with proteasome variant $\Delta 7 \mathrm{CP} \_\mathrm{HO}$ 3RA (right) was monitored by Coomassie-stained SDS-PAGE. The reaction was started with ATP and carried out at $37^{\circ} \mathrm{C}$. Aliquots were taken at the indicated time points and analyzed by SDS-PAGE. Representatives of three individual experiments are shown. Source data are provided as a Source Data file. $\mathbf{b}$ Time-resolved degradation of RecA during the recovery after mitomycin C (MMC)-induced DNA damage stress demonstrates the effect of a $\beta$ hairpin loop mutation of Mpa in M. smegmatis. RecA levels were monitored upon induction of MMC stress ( $80 \mathrm{ng} / \mathrm{mL}$ for $30 \mathrm{~min}$ ) and during the recovery phase in the M. smegmatis $\Delta$ mpa strain complemented with either WT Mpa or Mpa HLCg. To remove MMC, cells were washed ("wash") and then further incubated. RecA levels were analyzed by immunoblotting at the indicated time points. RpoB was probed in parallel as loading control. Representatives of three individual experiments are shown. Source Data are provided as a Source Data file.

pore loops contacting the substrate are shifted downwards by 2-3 Å compared to state A, which results in a translocation of the substrate towards the open gate of the proteasome by almost one amino acid.

The sequential cycles of substrate engagement and disengagement are tightly coupled with the nucleotide state of the ATPhydrolyzing motor ${ }^{41}$. The achieved resolution of Mpa allowed the identification of the bound nucleotide present in each protomer of Mpa (Supplementary Fig. 8). However, we can only differentiate between bound trinucleotide (ATP or ATP $\gamma \mathrm{S}$ ) and dinucleotide (ADP), but for the trinucleotide we cannot distinguish between ATP and ATP $\gamma$ S. Since the reaction was quenched with ATP $\gamma S$ but initially incubated with ATP (see Methods), both would in principle be a possibility. Therefore, ATP or ATP $\gamma \mathrm{S}$ will, for simplicity, be referred to as ATP.

The prominent position and strict conservation of lysine residue $\mathrm{K} 340$ in the translocation loop right adjacent to F341, suggests it plays a mechanistic role in the conformational cycle of loop movements and coupling to ATPase activity. In fact, K340 can form a salt-bridge with E344 from the neighboring protomer (Supplementary Fig. 9a), although this interaction is disrupted further down in the staircase, where the distance between the protomers increases. Interestingly, mutation of K340 to alanine abolishes the basal ATPase activity of Mpa on its own. This might point to a defect in the allosteric relay that stimulates the coordinated hydrolysis of ATP across the protomers in the Mpa ring. However, the ATPase activity is restored to $50 \%$ of wild-type level in the presence of both PupDHFR and proteasome (Supplementary Fig. 9b) and, in agreement with this, an alanine substitution variant, K340A, can still degrade PupDHFR model substrate in vitro, albeit at a mildly reduced rate (Supplementary Fig. 9c).

In state A, 5 of the 6 protomers of Mpa are in the ATP-bound state (Fig. 2a) with arginine fingers contributed by the neighboring subunit. The protomer with the pore loop at the bottom of the staircase (protomer 6, cyan) displays weak nucleotide density corresponding to the ADP nucleotide form. In this case, the arginine finger from the neighboring subunit is displaced and moved away from the bound nucleotide. There is also a prominent gap in the inter-subunit space between this protomer 
a

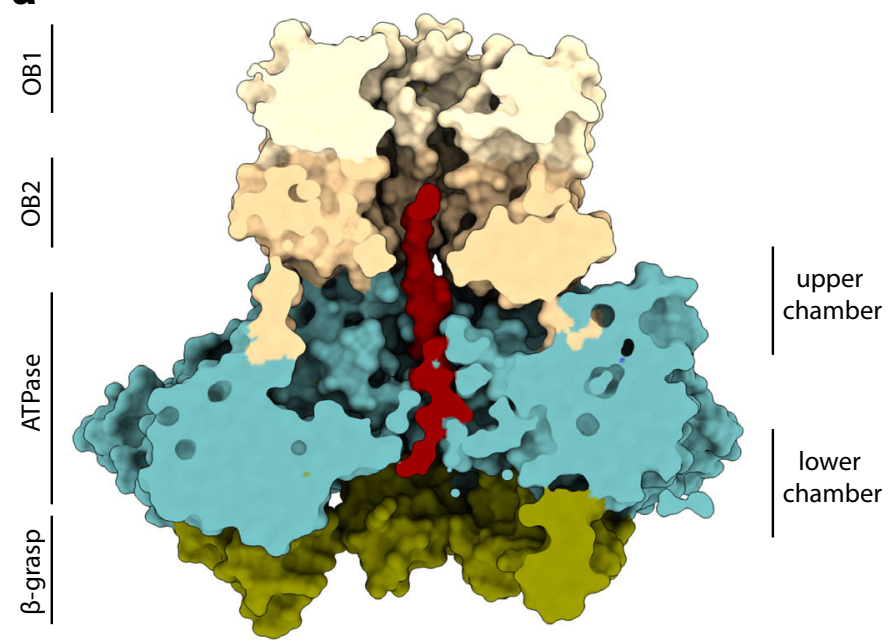

C

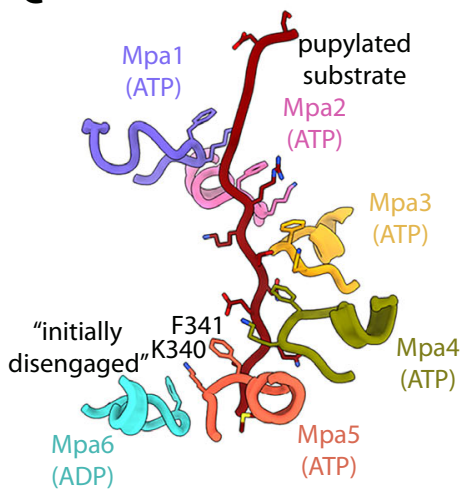

20S·Mpa.PupDHFR state $A$ d

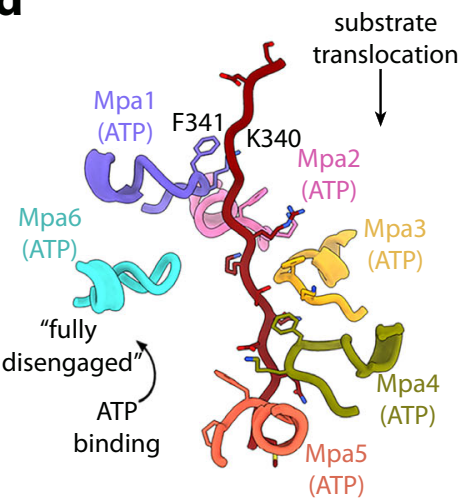

20S·Mpa.PupDHFR state $B$ b

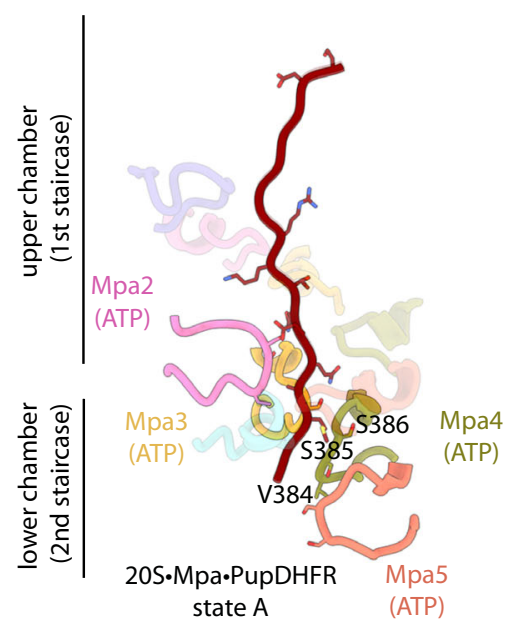

e

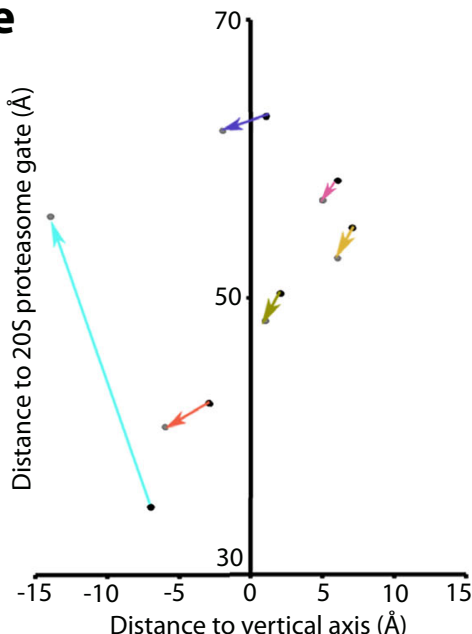

Fig. 5 Spiral-staircase arrangement of Mpa protomer loops contacting the engaged substrate in the ATPase chamber. a The cross-section of the Mpa hexamer with the substrate peptide chain (red) visualizes the shape of the inner channel and the location of Pup inside the channel. Pore loop 1 (K340E344) is located in the ATPase upper chamber and pore loop 2 (V384-S386) is located in the lower chamber. $\mathbf{b}$ Conformation of the pore loops in the ATPase lower chamber as resolved in state A is shown as cartoon with the contact residues (V384-S386) shown as sticks. The nucleotide state of the protomers is indicated. c Conformation of the pore loops in the ATPase chamber as resolved in state A is shown as cartoon with the contact residues (F341 and K340) shown as sticks. The nucleotide state of each protomer is indicated. d Conformation of the pore loops in the ATPase chamber as resolved in state B is shown as cartoon with the contact residues (F341 and K340) shown as sticks. The nucleotide state of each protomer is indicated. e Conformational changes in the pore loops as observed in state A and B are plotted based on the distance from the proteasome gate and distance from the vertical axis.

and the neighboring two Mpa protomers (Supplementary Fig. 8, left, bottom two panels). In state B in comparison, all protomers of Mpa are resolved with ATP bound, including protomer 6 (cyan) with its pore loop now in a disengaged and retracted conformation (Fig. 2b). Nevertheless, protomer 6 still exhibits less contacts to protomer 5 , and the arginine finger that it contributes to protomer 5 does not contact the nucleotide, thereby creating a fissure in the ATPase ring between these protomers.

\section{Discussion}

Actinobacteria acquired proteasomal genes and evolved a ubiquitin-like post-translational modification pathway to increase their survival under particularly challenging conditions, such as those encountered by $\mathrm{Mtb}$ inside host macrophages ${ }^{43}$. Centrally important to this pathway is the interaction of the AAA unfoldase Mpa with the 20S CP and the recruitment of protein substrates marked by pupylation for degradation by this molecular machine.
Despite the significance of this complex as a drug target 44 structural information on the fully assembled, active protease has been lacking. Our high-resolution cryo-EM structure of the substrate-engaged $\mathrm{Mpa}$-proteasome complex reveals the dynamic character of the interaction between the AAA unfoldase ring and the proteasome core cylinder. It also delivers a molecular understanding of how Pup, the functional analog of ubiquitin in proteasomal targeting, engages with the Mpa pore to initiate substrate processing.

In eukaryotic cells, the $26 \mathrm{~S}$ proteasome is responsible for the ATP-dependent turnover of protein substrates in the cytosol and nucleus. Together with the E1, E2, E3 enzyme cascade responsible for post-translational modification of target proteins with polyubiquitin chains, this recruitment and degradation pathway is referred to as the ubiquitin-proteasome system (UPS) ${ }^{4,45,46}$. The Pup-proteasome system (PPS) represents the bacterial functional analog of this degradation pathway ${ }^{47,48}$. The AAA unfoldase Mpa and the bacterial CP show homology to the eukaryotic regulatory 
ATPase subunits (Rpts) and the eukaryotic 20S, respectively. In contrast, bacterial ubiquitin-like targeting is an example of convergent evolution, and neither Pup and ubiquitin, nor the Pup ligase and the E1, E2, E3 cascade are homologous. Furthermore, the bacterial proteasomal unfoldase Mpa does not associate with additional non-ATPase subunits for substrate recruitment like it is the case for the eukaryotic 19S particle, where ubiquitin receptors Rpn1, Rpn10 and Rpn13 bind the polyubiquitin $\operatorname{tag}^{49-51}$. Instead, the intrinsically disordered Pup forms a long helix in the middle region of its primary structure (residues 22-50) that engages directly into an antiparallel, three-stranded coiled-coil with one of the N-terminal coiled-coil domains extending from the Mpa double-OB ring ${ }^{19,31}$. In addition to the poly-Ub tag, eukaryotic proteasome substrates additionally must feature an unstructured initiation region that can be threaded into the Rpt-ring pore for engagement by the translocation loops ${ }^{52,53}$, since Ub usually does not enter the ATPase motor, but is cleaved away by the deubiquitinase Rpn11 located close to the entrance pore $^{54}$. In contrast, Pup as recruitment tag brings along the determinant for pore engagement in the form of its intrinsically disordered $\mathrm{N}$-terminal region ${ }^{8,32}$.

Our structure trapped the Mpa-20S CP complex in the process of Pup-mediated substrate priming of the AAA motor. The $\mathrm{N}$-terminal methionine of Pup (M1) is located in the lower Mpa chamber about $35 \AA$ from the $20 \mathrm{~S}$ gate. The Mpa $\beta$-grasp domains form a sort of collar around the $20 \mathrm{~S}$ entrance gate, raising the AAA ring tier away from the $\alpha$-ring surface by about $15 \AA$, whereas in contrast the Rpts in the $26 \mathrm{~S}$ proteasome lie flat on the $\alpha$-rings. Inside Mpa, the 15 resolved N-terminal Pup residues traverse a distance of about $50 \AA$ from the second $\mathrm{OB}$ ring through the AAA ring-tier along the spiral-staircase. In the first $\mathrm{OB}$ ring, the density of substrate is not visible, but distance measurements show that Pup must span an additional $25 \AA$ from the last resolved residue to the rim of the first $\mathrm{OB}$ ring (the pore entrance), corresponding to about seven residues in extended conformation. This would suggest that Pup residue 22 is located at or close to the entrance pore, and Pup is likely still engaged in the shared three-stranded coil with the Mpa coiled-coil domains. In the next step, ATPase-driven Pup translocation through the spiral-staircase of the Mpa AAA motor toward the 20S gate must overcome this interaction to allow translocation of unfolded Pup and DHFR into the proteasomal degradation chamber. This might explain why Pup is arrested in the Mpa channel in the same register across the particle population. Furthermore, the observation that substrate is stalled at this stage might indicate that this step presents a notable energy barrier and constitutes a rate-limiting step in the reaction cycle.

Recent developments in single-particle cryo-electron microscopy have enabled the determination of high-resolution structures of numerous substrate-engaged AAA+ machines including proteasome holoenzymes, and a common mechanism of substrate translocation has emerged ${ }^{40,55-57}$. In agreement with other substrate-engaged AAA + ATPase structures, Mpa AAA motor pore loops adopt a spiral-staircase arrangement encircling the unfolded substrate, whereby the canonical aromatic F341 loop residue and the highly conserved K340 make the most prominent interactions with the polypeptide chain. It has been observed that a positively charged residue ( $\mathrm{K}$ or $\mathrm{R}$ ) frequently precedes the aromatic loop residue ${ }^{41}$. Across Actinobacteria the lysine in this position is strictly conserved, and a lysine is also found at this position in all but one of the Rpts of the eukaryotic proteasome. However, while the mutation of F341 is known to completely abolish substrate unfolding ${ }^{8}$, the K340A variant showed only a slightly lower rate of PupDHFR degradation. Interestingly, Mpa $\mathrm{K} 340 \mathrm{~A}$ in the absence of the $20 \mathrm{~S}$ proteasome has an order of magnitude lower ATPase activity, pointing to a potential defect in the allosteric coordination of activity across subunits in the ring. Nevertheless, this defect can be overcome and activity restored to $50 \%$ of wild-type activity in the presence of both substrate and proteasome. It is possible that this residue is particularly important in the context of proteasome-independent roles played by $\mathrm{Mpa}$, since the presence of substrate alone can only restore the activity to about $20 \%$ of wild-type activity. In C. glutamicum, for example, proteasomal subunits are absent, and Mpa was shown to be important for disassembly of ferritin during iron limitation ${ }^{58}$.

We resolved two distinct, sequential states of the Mpa complex with differences in spiral-staircase arrangement, nucleotide occupancy, and contacts between subunits and to the engaged substrate. Our observations agree with the increasing body of literature on substrate-bound structures of AAA + motors pointing to a sequential, hand-over-hand mechanism ${ }^{41}$. In state A, the bottom protomer is in the ADP-bound state and displays a pronounced gap to the preceding subunit in the spiral, indicating it is in the process of disengaging from the substrate. Upon exchange of ADP for ATP, in state B, this protomer has moved to a higher position and is now disengaged from the substrate that has moved by about one residue length toward the proteasomal gate. State B can be seen as an intermediate state, and to complete the step, the disengaged subunit has to move into the topmost position, re-engaging the substrate at the top of the staircase, thereby resulting again in state A but with the protomers having advanced by one position in the ring. The two states are also consistent with previously observed states in the cryo-EM structures of substrate-engaged $26 \mathrm{~S}$ proteasome ${ }^{40,57}$, Cdc48 46,59 , and $\mathrm{ClpXP} / \mathrm{AP}^{60-64}$ structures. Ultimately, successive rounds of substrate engagements/disengagements would allow for substrate translocation into the $\mathrm{CP}$ chamber, where it is degraded.

Our structure demonstrates that the $\beta$-grasp domain in the C-terminal region of Mpa adopts a conformation that allows the GQYL motif to dock into the pockets of the proteasomal $\alpha$-rings. The flexible anchoring of Mpa to the proteasome via the GQYL motif combined with the rail-like interactions between the $\beta$ hairpin loop and the $\mathrm{HO}$ helices of the $\mathrm{CP}$ a-rings solves the problem of maintaining the necessary affinity between Mpa and the proteasome, while at the same time allowing the full range of conformational changes in Mpa that are associated with substrate unfolding and translocation into the proteasomal degradation chamber. The functional significance of the Mpa $\beta$-hairpin loop in providing charge-complementary contacts along the radial rail system generated by the $\mathrm{H} 0$ helices of the $\mathrm{CP}$ a-rings is further supported by the impairment of substrate degradation in the presence of the Mtb Mpa variant, where the native sequence of the loop was replaced with the one from C. glutamicum. In agreement with the role in proteasome interaction that we describe, residues 501-505 (ANGDK) constituting the $\beta$-hairpin are well conserved in those members of Actinobacteria that possess a $20 \mathrm{~S}$ proteasome, but except for the central glycine important for the tight turn, the $\beta$-hairpin residues are not conserved in those species that lack proteasome core genes, like $C$. glutamicum (Supplementary Fig. 6).

The results presented here provide structural and mechanistic insights into the mycobacterial proteasome complex, revealing the molecular basis of the interaction between the hexameric AAA unfoldase Mpa and the proteasome core particle. Our study explains how the unfoldase and protease components can stably associate, while still allowing the AAA motor-mediated conformational changes required to unfold and translocate the substrate. Analogous mechanistic solutions may also exist in other chaperone-protease systems and may even be the reason for the evolutionarily conserved symmetry mismatch between the hexameric ATP-driven unfoldases and the heptameric proteolytic core particles, since the formation of symmetry-matched contacts 
between the two would likely interfere with the required conformational mobility of the AAA motor. Furthermore, our structure reveals how in mycobacteria pupylated substrates are engaged and translocated into the proteolytic chamber of the proteasome for degradation.

\section{Methods}

Generation of an unmarked $\boldsymbol{M}$. smegmatis mpa deletion strain. Regions of roughly $1500 \mathrm{bp}$ upstream and downstream of $m p a$ were amplified from $M$. smegmatis $\mathrm{MC}^{2} 155$ SMR5 genomic DNA by PCR (for primer sequences see Supplementary Table 2). Fragments were cloned into PCR-linearized p2NIL vector (p2NIL was a gift from Tanya Parish ${ }^{65}$; Addgene plasmid \#20188) by isothermal DNA assembly. The resulting plasmid was digested with PacI and ligated with the PacI-excised marker cassette (hygR, lacZ, and sacB) from pGOAL19 (pGOAL19 was a gift from Tanya Parish 65 ; Addgene plasmid \#20190) to yield the mpa suicide plasmid. Suicide plasmid $(1 \mu \mathrm{g})$ was UV-irradiated for $1 \mathrm{~min}$ before transformation into $300 \mu \mathrm{l}$ M. smegmatis MC 155 SMR5 cells by electroporation $(0.2 \mathrm{~cm}$ cuvette, $2.5 \mathrm{kV}$ pulse, BioRad GenePulser). Electroporated cells were immediately recovered in $6.5 \mathrm{ml} 7 \mathrm{H} 9$ shaking cultures for $6 \mathrm{~h}$ at $37^{\circ} \mathrm{C}$ after which $150 \mu \mathrm{l}$ culture were plated onto $7 \mathrm{H} 10$ agar supplemented with kanamycin $(25 \mu \mathrm{g} / \mathrm{ml})$ and hygromycin $(50 \mu \mathrm{g} / \mathrm{ml})$. Cells in the remaining culture volume were pelleted by centrifugation and plated as well. Colonies appeared after 3 days of incubation at $37^{\circ} \mathrm{C}$. Singlecrossover colonies (SCOs) were identified by X-gal underlay $(200 \mu \mathrm{l}$ of $0.4 \%(\mathrm{w} / \mathrm{v})$ $\mathrm{X}$-gal in DMSO were spread underneath the agar and plates were incubated overnight at $37^{\circ} \mathrm{C}$ ). Four positive SCOs (blue color) were picked into $5 \mathrm{ml}$ $7 \mathrm{H} 9$ supplemented with kanamycin $(25 \mu \mathrm{g} / \mathrm{ml})$ and hygromycin $(50 \mu \mathrm{g} / \mathrm{ml})$. SCO shaking cultures were grown at $37^{\circ} \mathrm{C}$ for 2 days before diluting an aliquot to OD600 of 0.8 and preparing a 10-fold dilution series in 7H9. $100 \mu \mathrm{l}$ of dilutions $1: 1$, $1: 10$, and $1: 100$ were plated on plain $7 \mathrm{H} 10$ agar and $7 \mathrm{H} 10$ agar supplemented with $2 \%(\mathrm{w} / \mathrm{v})$ sucrose. Plates were incubated at $37^{\circ} \mathrm{C}$ for 3 days and subsequently analyzed by X-gal underlay. Positive double-crossover colonies (DCOs; white color) were screened for mpa deletion by colony PCR, streaked on plain 7H10 agar and $7 \mathrm{H} 10$ supplemented with kanamycin $(25 \mu \mathrm{g} / \mathrm{ml})$ and grown at $37^{\circ} \mathrm{C}$.

Kanamycin-sensitive DCOs identified as bearing the mpa deletion in the colony PCR screen were further examined by amplification and sequencing of the $m p a$ genomic locus. Of the remaining candidates, one DCO was selected, grown in 7H9 liquid culture followed by preparation of glycerol stocks which were frozen in liquid nitrogen and stored at $-80^{\circ} \mathrm{C}$ until use. The absence of the Mpa protein in the cellular lysate of the newly generated knockout strain was further confirmed by immunoblotting.

Bacterial strains and culture conditions. Strains used were M. smegmatis $M^{2} 155$ SMR5 and MC ${ }^{2} 155$ SMR5 $\Delta m p a$. Bacteria were routinely grown in Middlebrook 7H9 (supplemented with $0.2 \% \mathrm{v} / \mathrm{v}$ glycerol and $0.05 \% \mathrm{w} / \mathrm{v}$ Tween-80) or on Middlebrook 7H10 agar plates. If applicable, hygromycin B (Carl Roth) was added to a final concentration of $50 \mu \mathrm{g} / \mathrm{mL}$. Liquid cultures were routinely grown at $37^{\circ} \mathrm{C}$ in a shaking incubator and agar plates were incubated at $37^{\circ} \mathrm{C}$ for growth.

Alignment. Protein sequence alignment was carried out using the Clustal X2.1 multiple sequence alignment software ${ }^{66}$. ESPript 3.0 was used to generate the graphical representation of the alignment ${ }^{67}$.

Cloning, expression and purification of proteins. The sequence of Mtb $m p a$ encoding the untagged, wild-type protein was previously cloned into pET20 (Novagen) via NdeI and BamHI restriction sites to generate the pET20-Mpa overexpression plasmid ${ }^{8}$. The plasmids coding for Mpa variants were obtained from pET20-Mpa by site-directed mutagenesis.

The plasmid for overexpression of PupDHFR was constructed by Gibson assembly (NEB) from the previously cloned pET20 plasmid coding for Mtb Pup with an N-terminal $\mathrm{His}_{10}$-Thioredoxin fusion (His $10^{-}$-Thioredoxin-MtbPupE) and the PCR fragment containing the E. coli dihydrofolate reductase gene. The sequences of primers used in this study are provided in Supplementary Table 2.

Sequences encoding Mtb $\Delta 7 \operatorname{PrcA}$ and $\mathrm{Mtb} \Delta 57 \operatorname{PrcB}$ ( $\alpha$-subunit is $\mathrm{N}$-terminally truncated by seven residues, $\beta$-subunit propeptide sequence is removed) were cloned into a single pET-Duet-1 (Novagen) overexpression plasmid with $\Delta 57 \operatorname{PrcB}$ forming a C-terminal fusion with the Strep-tag sequence to allow for simultaneous expression of both proteins and isolation of the assembled 20S CP by affinity purification. The plasmid coding for proteasome variant $\triangle 7 \mathrm{CP} \_$H0-3RA was generated from pET-Duet-1- $\Delta 7 \operatorname{PrcA}-\Delta 57 \operatorname{PrcB}$ by site-directed mutagenesis.

Mpa variants were expressed in E. coli Rosetta (DE3) cells at $30^{\circ} \mathrm{C}$ with IPTG induction $(0.1 \mathrm{mM}, 4 \mathrm{~h})$. Cells were harvested (F9S, $7000 \mathrm{rpm}(9180 \mathrm{~g}), 10 \mathrm{~min}$, $4{ }^{\circ} \mathrm{C}$ ), cell pellets were frozen with liquid $\mathrm{N}_{2}$ and stored at $-20^{\circ} \mathrm{C}$ until purification. Cell pellets were resuspended in buffer FFQ-A ( $50 \mathrm{mM}$ HEPES/KOH pH 7.5, $50 \mathrm{mM} \mathrm{NaCl}, 2 \mathrm{mM}$ EDTA, 10\% glycerol, $1 \mathrm{mM}$ DTT) supplemented with $1 \mathrm{X}$ PMSF (1 mM) and $1 \mathrm{X}$ cOmplete protease inhibitor (Roche), and the cells were lysed by high pressure shear force using a Microfluidizer M110-L device (Microfluidics; 5 passes, $11,000 \mathrm{psi}$ chamber pressure). After removal of cell debris (SS34, 20,000 rpm $\left.(47,810 \mathrm{~g}), 4^{\circ} \mathrm{C}, 1 \mathrm{~h}\right)$, cleared lysate was separated by anion- exchange chromatography (Fast Flow Q $65 \mathrm{~mL}$; buffer FFQ-A: see above; buffer FFQ-B: same as FFQ-A but $1 \mathrm{M} \mathrm{NaCl}$; gradient $8-60 \%$ of FFQ-B over $8 \mathrm{CV}$ ) and Mpa-containing fractions were pooled, precipitated with $60 \%\left(\mathrm{NH}_{4}\right)_{2} \mathrm{SO}_{4}$, redissolved, dialyzed and further purified by cation-exchange (Source $30 \mathrm{~S} 20 \mathrm{~mL}$ buffer 30S-A: $25 \mathrm{mM}$ MES/KOH pH 6.0, 10\% glycerol, $1 \mathrm{mM}$ DTT, $2 \mathrm{mM}$ EDTA buffer $30 \mathrm{~S}-\mathrm{B}$ : same as $30 \mathrm{~S}-\mathrm{A}$ but $1 \mathrm{M} \mathrm{NaCl}$; gradient $0-30 \%$ of $30 \mathrm{~S}-\mathrm{B}$ over $15 \mathrm{CV}$ ) and size-exclusion chromatography (Superose 6; $50 \mathrm{mM} \mathrm{HEPES/KOH} \mathrm{pH} \mathrm{7.5,}$ $150 \mathrm{mM} \mathrm{NaCl}, 10 \%$ (v/v) glycerol, $1 \mathrm{mM}$ EDTA, $1 \mathrm{mM}$ DTT). Mpa variants were stored at $-20^{\circ} \mathrm{C}$ in $50 \mathrm{mM}$ HEPES/KOH pH $7.5,150 \mathrm{mM} \mathrm{NaCl}, 10 \%$ (v/v) glycerol, $1 \mathrm{mM}$ EDTA, $1 \mathrm{mM}$ DTT.

Pup(E)DHFR was expressed in E. coli Tuner (DE3) cells at $37^{\circ} \mathrm{C}$ with IPTG induction $(0.2 \mathrm{mM})$ as $\mathrm{His}_{10}$-Thioredoxin-TEV-Pup(E)-DHFR fusion protein and purified by $\mathrm{Ni}^{2+}$-affinity chromatography. After cleavage of the fusion protein with TEV-protease, $\mathrm{Hi}_{10}$-Thioredoxin and TEV-protease were removed by $\mathrm{Ni}^{2+}$. affinity chromatography. Pup(E)DHFR was stored in $50 \mathrm{mM}$ Tris base/ $\mathrm{HCl} \mathrm{pH} \mathrm{7.5,}$ $150 \mathrm{mM} \mathrm{NaCl}, 1 \mathrm{mM}$ EDTA.

Wild type and H0-3RA variants of open-gate $20 \mathrm{~S}$ proteasome were expressed in E. coli Rosetta (DE3) cells at $25^{\circ} \mathrm{C}$ overnight with IPTG induction $(0.1 \mathrm{mM})$ and purified by affinity chromatography with StrepTactinXT resin (IBA Lifesciences) followed by size-exclusion chromatography (Superose 6). The purified open-gate proteasomes were stored at $4{ }^{\circ} \mathrm{C}$ in $50 \mathrm{mM}$ HEPES/KOH pH $7.54^{\circ} \mathrm{C}, 150 \mathrm{mM}$ $\mathrm{NaCl}, 1 \mathrm{mM}$ EDTA, $10 \%$ glycerol.

All protein concentrations were determined by absorbance spectroscopy at $280 \mathrm{~nm}$. Mpa concentrations are provided in terms of hexamers. Proteasome concentrations are given in terms of assembled core particle.

Proteasomal degradation assays. Open-gate proteasome variant $(0.2 \mu \mathrm{M}), \mathrm{Mpa}$ or Mpa variant $(0.2 \mu \mathrm{M})$ and PupDHFR $(2 \mu \mathrm{M})$, were mixed in buffer $\mathrm{R}(50 \mathrm{mM}$ HEPES/KOH pH $7.54^{\circ} \mathrm{C}, 150 \mathrm{mM} \mathrm{NaCl}, 5 \%$ glycerol, $10 \mathrm{mM} \mathrm{KCl}, 20 \mathrm{mM} \mathrm{MgCl}_{2}$, $1 \mathrm{mM}$ DTT). The reaction was started by the addition of ATP $(10 \mathrm{mM})$ in a total volume of $150 \mu \mathrm{L}$ and was carried out at $37^{\circ} \mathrm{C}$. Aliquots were collected at the indicated time points and analyzed by SDS-PAGE (sodium dodecyl sulfate-polyacrylamide gel electrophoresis). Experiments were repeated independently three times yielding similar results. Source Data (uncropped gels) are provided in the Source Data file.

\section{Complementation studies of $\boldsymbol{M}$. smegmatis mpa knockout strain and time-} resolved RecA degradation assay. M. smegmatis $\Delta m p a$ strain was complemented with either wild type or mutant Mpa under control of the native mpa promotor using modified pMyNT vectors containing the L5 integrase gene and grown in $100 \mathrm{~mL}$ shaking cultures at $37^{\circ} \mathrm{C}$ in $7 \mathrm{H} 9$ medium in the presence of hygromycin $\mathrm{B}$ $(50 \mu \mathrm{g} / \mathrm{mL})$. Cells were subjected to DNA damage stress by addition of mitomycin $\mathrm{C}(\mathrm{MMC}, 80 \mathrm{ng} / \mathrm{mL})$ at an OD600 of 1.0 to 1.2 . After $30 \mathrm{~min}$ incubation with MMC, cells were harvested by centrifugation $\left(3500 \mathrm{~g}, 10 \mathrm{~min}, 25^{\circ} \mathrm{C}\right)$, washed once in $100 \mathrm{~mL}$ fresh $7 \mathrm{H} 9$ medium, resuspended in $100 \mathrm{~mL}$ fresh $7 \mathrm{H} 9$ medium, and transferred to a new, sterile flask to ensure complete removal of MMC. Cultures were incubated for another $48 \mathrm{hr}, 5 \mathrm{~mL}$ aliquots were withdrawn at the indicated time points and cells were collected by centrifugation $\left(3500 \mathrm{~g}, 5 \mathrm{~min}, 4^{\circ} \mathrm{C}\right)$. Pellets were immediately flash-frozen in liquid $\mathrm{N}_{2}$ and stored at $-20^{\circ} \mathrm{C}$ for later analysis. Pellets were then resuspended in $800 \mu \mathrm{l}$ lysis buffer $(50 \mathrm{mM}$ HEPES/KOH pH 7.5, $150 \mathrm{mM} \mathrm{NaCl}, 1 \mathrm{mM}$ EDTA, $1 \mathrm{mM}$ PMSF, $1 \mathrm{X}$ cOmplete EDTA-free protease inhibitor (Roche) and lysed by bead beating in $2 \mathrm{ml}$ screw cap tubes containing $500-700 \mathrm{mg} 0.15 \mathrm{~mm}$ zirconium oxide beads using an MP Biomedicals FastPrep-24 bead beater $(3 \times 30 \mathrm{~s}, 6 \mathrm{~m} / \mathrm{s}, 1 \mathrm{~min}$ pause on ice in between). After removing insoluble material by centrifugation $\left(16,000 \mathrm{~g}, 10 \mathrm{~min}, 4^{\circ} \mathrm{C}\right)$, protein content of the cleared lysate was determined by Bradford assay. $5 \mu \mathrm{g}$ of total protein were separated on a Mini-PROTEAN stain-free $4-20 \%$ gradient polyacrylamide gel (BioRad) and transferred onto PVDF membrane Immobilon-P (Merck) using a BioRad Trans-Blot SD semidry transfer cell $\left(20 \mathrm{~V}\right.$, maximum of $\left.10 \mathrm{~mA} / \mathrm{cm}^{2}, 30 \mathrm{~min}\right)$ Membranes were blocked in PBS containing 0.05\% (v/v) Tween-20 and 5\% (w/v) nonfat dried milk for $1 \mathrm{~h}$ at room temperature. Immunoblotting for RecA and RpoB was carried out using commercially available anti-RecA antibody (1:2000, MBL International, clone ARM414) raised in mouse or commercially available anti-RpoB antibody (1:5000, BioLegend, clone 8RB13) raised in mouse ${ }^{68}$. The blocked membrane was incubated with primary antibodies in blocking buffer with $0.5 \%(\mathrm{w} / \mathrm{v})$ nonfat dried milk for $1 \mathrm{~h}$ at room temperature. Detection was achieved by incubation with horseradish peroxidase-conjugated anti-mouse IgG antibody (1:5,000, Promega \#W4021) in blocking buffer with $0.5 \%$ (w/v) nonfat dried milk for $1 \mathrm{~h}$ at room temperature and using ECL substrate (BioRad Clarity Western Substrate). Three complete, individual experiments were carried out, each starting from a single colony of freshly transformed and plated M. smegmatis $\Delta m p a$ cells, one representative is shown in Fig. $4 \mathrm{~b}$. Source Data (uncropped and unprocessed blots) are provided in the Source Data file.

ATPase activity of Mpa variants. ATPase activity of wild-type Mpa and Mpa variants was measured at $23^{\circ} \mathrm{C}$ in buffer $\mathrm{R}$ with a continuous spectrophotometric assay coupled to inorganic phosphate production using 7-methylinosine and the enzyme purine nucleoside phosphorylase ${ }^{69}$. The calibration curve was recorded in the presence of PNPase $(1.4 \mathrm{U}), 7$-methylinosine $(1 \mathrm{mM})$ and $0-600 \mu \mathrm{M} \mathrm{K}_{2} \mathrm{HPO}_{4}$. 
The absorbance was monitored at $291 \mathrm{~nm}$ until the plateau was reached and the $A_{291}$ value at the plateau was recorded for each phosphate concentration and used to construct the calibration curve. The reaction contained Mpa variant $(0.1 \mu \mathrm{M})$, PNPase (1.4 U), 7-methylinosine (1 mM) and was started by addition of ATP $(10 \mathrm{mM})$. The absorbance was monitored at $291 \mathrm{~nm}$, the linear part of the curve was used to derive the ATPase activity. The ATPase activity of the Mpa K340A variant was additionally measured in the presence of open-gate proteasome $(0.1 \mu \mathrm{M})$, PupDHFR $(1 \mu \mathrm{M})$ or both open-gate proteasome and PupDHFR. The plots showing the measured ATPase activities were generated with GraphPad Prism 7.04.

EM specimen preparation and imaging. Open gate proteasome $(3 \mu \mathrm{M}), \mathrm{Mpa}$ $(3 \mu \mathrm{M})$, ATP $(5 \mathrm{mM}), 2 \mathrm{x}$ reaction buffer $\mathrm{R}$ and $\mathrm{ddH}_{2} \mathrm{O}$ were mixed in a total volume of $400 \mu \mathrm{L}$ and incubated at $37^{\circ} \mathrm{C}$ for $2 \mathrm{~min}$. The sample was then loaded onto a Superose 6 10-300GL gel filtration column pre-equilibrated in buffer $\mathrm{R}$ without glycerol at $4{ }^{\circ} \mathrm{C}$. The same buffer was used as running buffer. The elution fraction containing the $\mathrm{Mpa}-20 \mathrm{~S} \mathrm{CP}$ complex was concentrated using a $0.5 \mathrm{~mL}$ Amicon spin filter ( $100 \mathrm{kDa}$ MWCO) to approximately $2 \mathrm{mg} / \mathrm{mL}$.

Mpa-proteasome sample $(1.09 \mathrm{mg} / \mathrm{mL})$, PupDHFR $(1.06 \mathrm{mg} / \mathrm{mL})$ and ATP $(1.76 \mathrm{mM})$ were mixed together and incubated for $60 \mathrm{~s}$ on ice. ATP $\gamma \mathrm{S}(5.4 \mathrm{mM})$ and NP-40 $(0.003 \%)$ were added and the resulting sample was immediately applied on the grid.

Sample of the assembled complex $(5 \mu \mathrm{L})$ was applied to holey carbon R2/1 Quantifoil grids that were glow discharged with a Pelco EasyGlow system for $30 \mathrm{~s}$ prior to use. Grids were immediately blotted with filter paper and then plunged into liquid ethane/propane mix cooled to liquid nitrogen temperature using a ThermoFisher Vitrobot at $4{ }^{\circ} \mathrm{C}$ with $95 \%$ relative humidity. Data collection was performed on a Titan Krios electron microscope (ThermoFisher) operated at $300 \mathrm{kV}$, using the EPU software (ThermoFisher) for automated data acquisition in counting mode using the Gatan K3 direct electron detector with an energy filter slit width of $20 \mathrm{eV}$. Data were collected at a defocus of -1.2 to $-2.5 \mu \mathrm{m}$ range with step size of $0.1 \mu \mathrm{m}$ and at a nominal magnification of $\times 105,000$, which resulted in a calibrated pixel size of $0.84 \AA$ /pixel $(0.42 \AA /$ pixel in super-resolution mode). Illumination conditions were adjusted to an exposure rate of $32 \mathrm{e}-/ \mathrm{pixel} / \mathrm{second}$. Micrographs were recorded as movie stacks with an electron dose of $\sim 50$ electrons/ $\AA^{2}$ fractionated into a total of 40 frames. Drift and bright gain reference corrections in addition to dose-weighting were performed with MotionCor $2^{70}$.

Cryo-EM data processing and refinement. Contrast transfer function (CTF) was calculated using GCTF ${ }^{71}$ and the power spectra of the micrographs were then carefully inspected for drift, and images with signal extending to less than $5 \AA$ were discarded from the two datasets. Particle images $(860,718)$ were selected using the Laplacian Gaussian Blob as a reference as implemented in RELION3 ${ }^{72}$ from a total of 12,673 micrographs.

Initial processing of the images was done with CryoSPARC ${ }^{73}$. First, twodimensional (2D) image classification was performed on binned images. An abinitio refinement was then performed on selected particles $(385,394)$ from $2 \mathrm{D}$ classaverages that resolved features of the proteasome, asking for five initial starting models. The initial model that depicted a capped proteasome was then used as a reference for a heterogeneous 3D refinement in CryoSPARC asking for six 3D classes. Particles $(222,719)$ in the 3D class which displayed the capped 20S CP were selected. Other classes showing empty 20S CP or weak Mpa densities were discarded. To improve the local resolution of Mpa, the signal of the 20S CP was subtracted from each image, and aligned Mpa particles were subjected to 3D classification in RELION3. The particles in the two classes with the best-resolved features of Mpa were refined using signal-subtracted images in RELION3. To generate the 20S CP subtracted images, a soft mask was generated based on the Mpa density, which allowed the density of the 20S CP to be subtracted from individual particles and then the subtracted images to be re-centered on the center of mass of Mpa in RELION3. The center of mass was determined on masked Mpa by using the "shift_com" option using the relion_image_handler command ${ }^{72}$ resulting in shift values $x=-0.6, y=-2.6$, and $z=-78$ relative to the center of the box. Re-centered and subtracted images were then refined using a masked Mpa obtained from the 3D classification as an initial reference. The resulting maps of Mpa state A and state B, refined to 3.8 and $3.9 \AA$ resolution, were shifted back to the original position before re-centering. Validation of the relative positions was carried out by rigid body fitting of the focused Mpa maps into the globally refined map of the Mpa-20S CP, which only resulted in minor shifts in the individual focused maps without any rotations or tilts. Composite maps of Mpa and 20S CP were then generated by using the "add" option. To obtain a reconstruction of the $20 \mathrm{~S} \mathrm{CP}$, a tight mask was applied on the $20 \mathrm{~S} \mathrm{CP}$ and particles were $3 \mathrm{D}$ refined in RELION3, which resulted in a final reconstruction of $2.8 \AA$ resolution. All final 3D refinements and map sharpening were performed in RELION with a binned pixel size of $1.22 \AA$ /pixel. Local resolution and gold standard FSC plots using $\mathrm{FSC}=0.143$ as a criterion were calculated in RELION3 (Supplementary Fig. 2).

Model building in the cryo-EM maps. For model building into the cryo-EM map, the coordinates of mycobacterial 20S CP (PDB ID:5LZP [https://doi.org/10.2210/ $\mathrm{pdb} 5 \mathrm{LZP} / \mathrm{pdb}]^{74}$ ) were docked as rigid body into the cryo-EM map using USCF
CHIMERA, and adjusted based on their side chain/secondary structure densities. The GQYL-motif residues were built into their corresponding EM densities and adjusted in $\mathrm{COOT}^{75}$. For model building into the cryo-EM maps of Mpa states A and B, coordinates of one Mpa protomer (PDB ID: 5KWA [https://doi.org/ $10.2210 / \mathrm{pdb} 5 \mathrm{KWA} / \mathrm{pdb}]^{28}$ ) were used as a starting model and then divided into the OB ring, ATPase, helical, and $\beta$-grasp domains and docked as rigid bodies into each of the 6 protomers in states A and B. The coordinates were then adjusted based on the visible secondary structure elements and side-chain EM densities. The ATPase pore loops, which contact the substrate, were built de novo into the corresponding cryo-EM densities of both states A and B. The bound ATP or ADP nucleotides were placed based on their visible densities in the cryo-EM maps. The EM-density corresponding to the N-terminus of Pup was built de novo based on the visible side-chain density in COOT. To generate a full model for the 20S CP-Mpa complex, a composite EM map was generated by adding the 20S CP and Mpa EM maps from the focused 3D refinements in RELION. The models of Mpa state A or B and of the 20S CP were then docked as rigid bodies. For model refinements, all resulting models were then refined into the corresponding EM densities and subjected to eight cycles of real space refinements using phenix.real_space_refine in PHENIX ${ }^{76}$, during which protein secondary structure, Ramachandran and side-chain rotamer restraints were applied. The fit of the EM map was validated using the real space correlation coefficients (CCmask) between the model versus the map Fourier Shell Correlation (FSC) at FSC $=0.5$ as a cut-off criterion, which resulted in similar resolution as the half-set map FSC using $\mathrm{FSC}=0.143$ as a cut-off criterion. Images were prepared in either ChimeraX ${ }^{77}$ or $\mathrm{PyMOL}^{78}$.

Reporting summary. Further information on research design is available in the Nature Research Reporting Summary linked to this article.

\section{Data availability}

Protein structure data and the coordinates generated in this study have been deposited in the Protein Data Bank with PDB accession codes 7PX9 (Substrate-engaged Mpa in state A-focused 3D refinement), 7PXA (open-gate 20S CP-global 3D refinement), 7PXB (Substrate-engaged Mpa in state B-focused 3D refinement), 7PXC (Substrate-engaged $\mathrm{Mpa}$ in state A in complex with open-gate 20S $\mathrm{CP}-$ composite map) and 7PXD (Substrate-engaged Mpa in state B in complex with open-gate 20S CP-composite map) Cryo-EM maps have been deposited with the Electron Microscopy Data Bank as EMD13694 (Substrate-engaged Mpa in state A-focused 3D refinement), EMD-13695 (opengate 20S CP-global 3D refinement), EMD-13696 (Substrate-engaged Mpa in state Bfocused 3D refinement), EMD-13697 (Substrate-engaged Mpa in state A in complex with open-gate 20S CP-composite map) and EMD-13698 (Substrate-engaged Mpa in state $\mathrm{B}$ in complex with open-gate $20 \mathrm{~S} \mathrm{CP}$-composite map). Source data are provided with this paper.

Received: 25 June 2021; Accepted: 13 December 2021; Published online: 12 January 2022

\section{References}

1. Pohl, C. \& Dikic, I. Cellular quality control by the ubiquitin-proteasome system and autophagy. Science 366, 818-822 (2019).

2. Schramm, F. D., Schroeder, K. \& Jonas, K. Protein aggregation in bacteria. FEMS Microbiol Rev. 44, 54-72 (2020).

3. Yedidi, R. S., Wendler, P. \& Enenkel, C. AAA-ATPases in protein degradation. Front Mol. Biosci. 4, 42 (2017).

4. Bard, J. A. M. et al. Structure and function of the $26 \mathrm{~S}$ proteasome. Annu. Rev Biochem. 87, 697-724 (2018).

5. Laederach, J., Leodolter, J., Warweg, J. \& Weber-Ban, E. Chaperone-Proteases of Mycobacteria. in The Molecular Chaperones Interaction Networks in Protein Folding and Degradation (ed. Houry, W. A.) 419-444 (Springer, 2014).

6. Alhuwaider, A. A. H. \& Dougan, D. A. AAA + machines of protein destruction in mycobacteria. Front Mol. Biosci. 4, 49 (2017).

7. Wang, T. et al. Structural insights on the Mycobacterium tuberculosis proteasomal ATPase Mpa. Structure 17, 1377-1385 (2009).

8. Striebel, F., Hunkeler, M., Summer, H. \& Weber-Ban, E. The mycobacterial Mpa-proteasome unfolds and degrades pupylated substrates by engaging Pup's N-terminus. EMBO 29, 1262-1271 (2010).

9. Wolf, S. et al. Characterization of ARC, a divergent member of the AAA ATPase family from Rhodococcus erythropolis. J. Mol. Biol. 277, 13-25 (1998)

10. Darwin, K. H., Lin, G., Chen, Z., Li, H. \& Nathan, C. F. Characterization of a Mycobacterium tuberculosis proteasomal ATPase homologue. Mol. Microbiol. 55, 561-571 (2005)

11. Pearce, M. J., Mintseris, J., Ferreyra, J., Gygi, S. P. \& Darwin, K. H. Ubiquitinlike protein involved in the proteasome pathway of Mycobacterium tuberculosis. Science 322, 1104-1107 (2008). 
12. Burns, K. E., Liu, W. T., Boshoff, H. I., Dorrestein, P. C. \& Barry, C. E. 3rd Proteasomal protein degradation in Mycobacteria is dependent upon a prokaryotic ubiquitin-like protein. J. Biol. Chem. 284, 3069-3075 (2009).

13. Striebel, F. et al. Bacterial ubiquitin-like modifier Pup is deamidated and conjugated to substrates by distinct but homologous enzymes. Nat. Struct. Mol. Biol. 16, 647-651 (2009).

14. Guth, E., Thommen, M. \& Weber-Ban, E. Mycobacterial ubiquitin-like protein ligase PafA follows a two-step reaction pathway with a phosphorylated pup intermediate. J. Biol. Chem. 286, 4412-4419 (2011).

15. Sutter, M., Damberger, F. F., Imkamp, F., Allain, F. H. \& Weber-Ban, E. Prokaryotic ubiquitin-like protein (Pup) is coupled to substrates via the side chain of its C-terminal glutamate. J. Am. Chem. Soc. 132, 5610-5612 (2010).

16. Imkamp, F. et al. Dop functions as a depupylase in the prokaryotic ubiquitinlike modification pathway. EMBO Rep. 11, 791-797 (2010).

17. Burns, K. E. et al. "Depupylation" of prokaryotic ubiquitin-like protein from mycobacterial proteasome substrates. Mol. Cell 39, 821-827 (2010).

18. Chen, X. et al. Prokaryotic ubiquitin-like protein pup is intrinsically disordered. J. Mol. Biol. 392, 208-217 (2009).

19. Sutter, M., Striebel, F., Damberger, F. F., Allain, F. H. T. \& Weber-Ban, E. A distinct structural region of the prokaryotic ubiquitin-like protein (Pup) is recognized by the $\mathrm{N}$-terminal domain of the proteasomal ATPase Mpa. FEBS Lett. 583, 3151-3157 (2009).

20. Liao, S. et al. Pup, a prokaryotic ubiquitin-like protein, is an intrinsically disordered protein. Biochem J. 422, 207-215 (2009).

21. Barandun, J. et al. Prokaryotic ubiquitin-like protein remains intrinsically disordered when covalently attached to proteasomal target proteins. BMC Struct. Biol. 17, 1 (2017).

22. Barandun, J., Delley, C. L., Ban, N. \& Weber-Ban, E. Crystal structure of the complex between prokaryotic ubiquitin-like protein and its ligase PafA. J. Am. Chem. Soc. 135, 6794-6797 (2013).

23. Samanovic, M. I. et al. Proteasomal control of cytokinin synthesis protects Mycobacterium tuberculosis against nitric oxide. Mol. Cell 57, 984-994 (2015).

24. Elharar, Y. et al. Survival of mycobacteria depends on proteasome-mediated amino acid recycling under nutrient limitation. EMBO J. 33, 1802-1814 (2014).

25. Müller, A. U., Imkamp, F. \& Weber-Ban, E. The mycobacterial LexA/RecAindependent DNA damage response is controlled by PafBC and the pupproteasome system. Cell Rep. 23, 3551-3564 (2018)

26. Fascellaro, G. et al. Comprehensive proteomic analysis of nitrogen-starved mycobacterium smegmatis deltapup reveals the impact of pupylation on nitrogen stress response. J. Proteome Res. 15, 2812-2825 (2016).

27. Darwin, K. H., Ehrt, S., Gutierrez-Ramos, J. C., Weich, N. \& Nathan, C. F. The proteasome of Mycobacterium tuberculosis is required for resistance to nitric oxide. Science 302, 1963-1966 (2003).

28. Wu, Y. et al. Mycobacterium tuberculosis proteasomal ATPase Mpa has a beta-grasp domain that hinders docking with the proteasome core protease. Mol. Microbiol. 105, 227-241 (2017).

29. Lander, G. C. et al. Complete subunit architecture of the proteasome regulatory particle. Nature 482, 186-191 (2012).

30. Beck, F. et al. Near-atomic resolution structural model of the yeast $26 \mathrm{~S}$ proteasome. Proc. Natl Acad. Sci. USA 109, 14870-14875 (2012).

31. Wang, T., Darwin, K. H. \& Li, H. Binding-induced folding of prokaryotic ubiquitin-like protein on the Mycobacterium proteasomal ATPase targets substrates for degradation. Nat. Struct. Mol. Biol. 17, 1352-1357 (2010).

32. Burns, K. E., Pearce, M. J. \& Darwin, K. H. Prokaryotic ubiquitin-like protein provides a two-part degron to Mycobacterium proteasome substrates. $J$. Bacteriol. 192, 2933-2935 (2010).

33. Pearce, M. J. et al. Identification of substrates of the Mycobacterium tuberculosis proteasome. EMBO J. 25, 5423-5432 (2006).

34. Rabl, J. et al. Mechanism of gate opening in the $20 \mathrm{~S}$ proteasome by the proteasomal ATPases. Mol. Cell 30, 360-368 (2008).

35. Smith, D. M. et al. Docking of the proteasomal ATPases' carboxyl termini in the 20 S proteasome's alpha ring opens the gate for substrate entry. Mol. Cell 27, 731-744 (2007).

36. Lin, G. et al. Mycobacterium tuberculosis prcBA genes encode a gated proteasome with broad oligopeptide specificity. Mol. Microbiol. 59, 1405-1416 (2006).

37. Yin, Y., Kovach, A., Hsu, H. C., Darwin, K. H. \& Li, H. The mycobacterial proteasomal ATPase Mpa forms a gapped ring to engage the $20 \mathrm{~S}$ proteasome. J. Biol. Chem. 296, 100713 (2021).

38. Gandotra, S., Schnappinger, D., Monteleone, M., Hillen, W. \& Ehrt, S. In vivo gene silencing identifies the Mycobacterium tuberculosis proteasome as essential for the bacteria to persist in mice. Nat. Med. 13, 1515-1520 (2007).

39. WHO. Global Tuberculosis Report 2020 (World Health Organization, 2020).

40. Dong, Y. et al. Cryo-EM structures and dynamics of substrate-engaged human 26S proteasome. Nature 565, 49-55 (2019).
41. Gates, S. N. \& Martin, A. Stairway to translocation: AAA + motor structures reveal the mechanisms of ATP-dependent substrate translocation. Protein Sci. 29, 407-419 (2020)

42. Hryc, C. F. et al. Accurate model annotation of a near-atomic resolution cryoEM map. Proc. Natl Acad. Sci. USA 114, 3103-3108 (2017)

43. von Rosen, T., Keller, L. M. L. \& Weber-Ban, E. Survival in hostile conditions pupylation and the proteasome in actinobacterial stress response pathways. Front. Mol. Biosci. 8, 685757 (2021).

44. Zhang, H. et al. Macrocyclic peptides that selectively inhibit the Mycobacterium tuberculosis proteasome. J. Med. Chem. 64, 6262-6272 (2021).

45. Davis, C., Spaller, B. L. \& Matouschek, A. Mechanisms of substrate recognition by the $26 \mathrm{~S}$ proteasome. Curr. Opin. Struct. Biol. 67, 161-169 (2021).

46. Komander, D. \& Rape, M. The ubiquitin code. Annu. Rev. Biochem 81, 203-229 (2012).

47. Muller, A. U. \& Weber-Ban, E. The bacterial proteasome at the core of diverse degradation pathways. Front Mol. Biosci. 6, 23 (2019).

48. Becker, S. H. \& Darwin, K. H. Bacterial proteasomes: mechanistic and functional insights. Microbiol. Mol. Biol. Rev. 81, e00036-16 (2017).

49. Elsasser, S., Chandler-Militello, D., Muller, B., Hanna, J. \& Finley, D. Rad23 and Rpn10 serve as alternative ubiquitin receptors for the proteasome. J. Biol. Chem. 279, 26817-26822 (2004).

50. Husnjak, K. et al. Proteasome subunit Rpn13 is a novel ubiquitin receptor. Nature 453, 481-488 (2008)

51. Shi, Y. et al. Rpn1 provides adjacent receptor sites for substrate binding and deubiquitination by the proteasome. Science 351, aad9421 (2016).

52. Tomita, T. \& Matouschek, A. Substrate selection by the proteasome through initiation regions. Protein Sci. 28, 1222-1232 (2019).

53. Prakash, S., Tian, L., Ratliff, K. S., Lehotzky, R. E. \& Matouschek, A. An unstructured initiation site is required for efficient proteasome-mediated degradation. Nat. Struct. Mol. Biol. 11, 830-837 (2004).

54. Verma, R. et al. Role of Rpn11 metalloprotease in deubiquitination and degradation by the 26 S proteasome. Science 298, 611-615 (2002).

55. Rizo, A. N. et al. Structural basis for substrate gripping and translocation by the ClpB AAA+ disaggregase. Nat. Commun. 10, 2393 (2019).

56. Cooney, I. et al. Structure of the Cdc48 segregase in the act of unfolding an authentic substrate. Science 365, 502-505 (2019).

57. de la Peña, A. H., Goodall, E. A., Gates, S. N., Lander, G. C. \& Martin, A. Substrate-engaged $26 \mathrm{~S}$ proteasome structures reveal mechanisms for ATPhydrolysis-driven translocation. Science 362, eaav0725 (2018).

58. Küberl, A., Polen, T. \& Bott, M. The pupylation machinery is involved in iron homeostasis by targeting the iron storage protein ferritin. Proc. Natl Acad. Sci. USA 113, 4806-4811 (2016)

59. Twomey, E. C. et al. Substrate processing by the Cdc48 ATPase complex is initiated by ubiquitin unfolding. Science 365, eaax1033 (2019).

60. Gatsogiannis, C., Balogh, D., Merino, F., Sieber, S. A. \& Raunser, S. Cryo-EM structure of the ClpXP protein degradation machinery. Nat. Struct. Mol. Biol. 26, 946-954 (2019).

61. Fei, X. et al. Structures of the ATP-fueled ClpXP proteolytic machine bound to protein substrate. Elife 9, e52774 (2020).

62. Kim, S., Zuromski, K. L., Bell, T. A., Sauer, R. T. \& Baker, T. A. ClpAP proteolysis does not require rotation of the ClpA unfoldase relative to ClpP. Elife 9, e61451 (2020).

63. Lopez, K. E. et al. Conformational plasticity of the ClpAP AAA + protease couples protein unfolding and proteolysis. Nat. Struct. Mol. Biol. 27, 406-416 (2020).

64. Ripstein, Z. A., Vahidi, S., Houry, W. A., Rubinstein, J. L. \& Kay, L. E. A processive rotary mechanism couples substrate unfolding and proteolysis in the ClpXP degradation machinery. Elife 9, e52158 (2020).

65. Parish, T. \& Stoker, N. G. Use of a flexible cassette method to generate a double unmarked Mycobacterium tuberculosis tlyA plcABC mutant by gene replacement. Microbiol. (Read.) 146, 1969-1975 (2000).

66. Larkin, M. A. et al. Clustal W and Clustal X version 2.0. Bioinformatics 23, 2947-2948 (2007).

67. Robert, X. \& Gouet, P. Deciphering key features in protein structures with the new ENDscript server. Nucleic Acids Res. 42, W320-W324 (2014).

68. Fudrini Olivencia, B. et al. Mycobacterium smegmatis PafBC is involved in regulation of DNA damage response. Sci. Rep. 7, 13987 (2017)

69. Rieger, C. E., Lee, J. \& Turnbull, J. L. A continuous spectrophotometric assay for aspartate transcarbamylase and ATPases. Anal. Biochem. 246, 86-95 (1997).

70. Zheng, S. Q. et al. MotionCor2: anisotropic correction of beam-induced motion for improved cryo-electron microscopy. Nat. Methods 14, 331-332 (2017).

71. Zhang, K. Gctf: real-time CTF determination and correction. J. Struct. Biol. 193, 1-12 (2016). 
72. Zivanov, J. et al. New tools for automated high-resolution cryo-EM structure determination in RELION-3. eLife 7, e42166 (2018).

73. Punjani, A., Rubinstein, J. L., Fleet, D. J. \& Brubaker, M. A. cryoSPARC: algorithms for rapid unsupervised cryo-EM structure determination. Nat. Methods 14, 290-296 (2017).

74. Bolten, M. et al. Structural analysis of the bacterial proteasome activator Bpa in complex with the 20S proteasome. Structure 24, 2138-2151 (2016).

75. Emsley, P. \& Cowtan, K. Coot: model-building tools for molecular graphics. Acta Crystallogr. D: Biol. Crystallogr. 60, 2126-2132 (2004)

76. Adams, P. D. et al. PHENIX: a comprehensive Python-based system for macromolecular structure solution. Acta Crystallogr. D 66, 213-221 (2010).

77. Goddard, T. D. et al. UCSF ChimeraX: meeting modern challenges in visualization and analysis. J. Computational Chem. 27, 14-25 (2018).

78. Schrodinger, L. L. C. The PyMOL Molecular Graphics System Version 1.8 (Science and Education Publisher, 2015).

\section{Acknowledgements}

The authors thank A. Scaiola for support with EM data collection, M. Leibundgut for support with model building, and N. Ban for discussions. Cryo-EM data were collected at the Scientific Center for Optical and Electron Microscopy at the ETH Zurich (ScopeM). We thank the ScopeM staff for technical support during cryo-EM data collection. We gratefully acknowledge the support of NVIDIA Corporation for the Titan Xp GPU used in this research through a GPU Grant program awarded to A.J. This work was supported by a Swiss National Science Foundation (SNSF) grant to EWB (310030_185250).

\section{Author contributions}

M.K., A.J., and E.W.B. conceived and planned the study. M.K. cloned and purified all constructs and performed the biochemical experiments and complementation assays. M.K. and A.J. assembled the complex for cryo-EM analysis, prepared and screened the EM grids. A.J. collected the EM data and determined the cryo-EM structures. A.M. generated the M. smegmatis mpa knockout strain. M.K., A.J., and E.W.B. interpreted the results and wrote the manuscript.

\section{Competing interests}

The authors declare no competing interests.

\section{Additional information}

Supplementary information The online version contains supplementary material available at https://doi.org/10.1038/s41467-021-27787-3.

Correspondence and requests for materials should be addressed to Ahmad Jomaa or Eilika Weber-Ban.

Peer review information Nature Communications thanks Paula da Fonseca, Youdong Mao and the other, anonymous, reviewer for their contribution to the peer review of this work.

Reprints and permission information is available at http://www.nature.com/reprints

Publisher's note Springer Nature remains neutral with regard to jurisdictional claims in published maps and institutional affiliations.

Open Access This article is licensed under a Creative Commons Attribution 4.0 International License, which permits use, sharing, adaptation, distribution and reproduction in any medium or format, as long as you give appropriate credit to the original author(s) and the source, provide a link to the Creative Commons license, and indicate if changes were made. The images or other third party material in this article are included in the article's Creative Commons license, unless indicated otherwise in a credit line to the material. If material is not included in the article's Creative Commons license and your intended use is not permitted by statutory regulation or exceeds the permitted use, you will need to obtain permission directly from the copyright holder. To view a copy of this license, visit http://creativecommons.org/ licenses/by/4.0/.

(C) The Author(s) 2022 